\title{
Remarks on highly supersymmetric backgrounds of 11-dimensional supergravity
}

\author{
Andrea Santi
}

\begin{abstract}
This note focuses on some properties and uses of filtered deformations in the context of $D=11$ supergravity. We define the concept of abstract symbol and give a strong version of the Reconstruction Theorem, namely a bijective correspondence from the space of highly supersymmetric supergravity backgrounds to the space of abstract symbols. We propose a general strategy to construct highly supersymmetric supergravity backgrounds and present an example in detail, which includes the computation of the ideal generated by the Killing spinors of two known pp-wave backgrounds with $N=24$ supersymmetry. Finally, we give an alternative proof, based on the isotropy algebra of a supergravity background, of a classical supersymmetry gap result of Gran, Gutowski, Papadopoulos and Roest.
\end{abstract}

\section{Introduction}

An important line of research in string and M-theory is the construction of backgrounds of their low-energy effective counterparts, i.e., supergravity, since this is where strings and higher-dimensional branes can propagate. Backgrounds preserving maximal or near to maximal supersymmetry are important classes of such solutions.

In particular, the classical limit of M-theory is supergravity in $D=11$ dimensions. From a geometric perspective, a background of $D=11$ supergravity consists of an 11-dimensional Lorentzian spin manifold $(M, g)$ with a closed 4-form $F \in \Omega^{4}(M)$, satisfying the following coupled system of PDEs:

$$
\begin{aligned}
d \star F & =\frac{1}{2} F \wedge F, \\
\operatorname{Ric}(X, Y) & =\frac{1}{2} g\left(l_{X} F, l_{Y} F\right)-\frac{1}{6}\|F\|^{2} g(X, Y),
\end{aligned}
$$

Andrea Santi

University of Padova, Mathematics Department “Tullio Levi-Civita”, 35121 Padova (Italy), e-mail: asanti.math@gmail.com 
for all $X, Y \in \mathfrak{X}(M)$. These are the bosonic field equations of $D=11$ supergravity.

Let $S(M) \rightarrow M$ be the spinor bundle of $(M, g)$, with typical fiber the irreducible module $S \cong \mathbb{R}^{32}$ of the Clifford algebra $C \ell(V) \cong 2 \mathbb{R}(32)$. A spinor field $\varepsilon \in \Gamma(S(M))$ is called a Killing spinor if we have

$$
\nabla_{X} \varepsilon-\frac{1}{24}(X \cdot F-3 F \cdot X) \cdot \varepsilon=0
$$

for all $X \in \mathfrak{X}(M)$, where $\nabla$ is the Levi-Civita connection and - Clifford multiplication. The connection on $S(M)$ defined by the L.H.S. of (2) is called the "superconnection" and the amount of spinors it preserves, i.e., Killing spinors, is an important invariant of a supergravity background.

Backgrounds with $16<N \leq 32$ Killing spinors are called highly supersymmetric and their classification is widely open. Although this might be a distant goal, a number of different solutions have been proposed in the literature:

- Maximally supersymmetric backgrounds, i.e., with $N=32$ Killing spinors,

- pp-waves backgrounds, i.e., Brinkmann spaces with flat transverse geometry,

- Gödel backgrounds, i.e., solutions admitting closed timelike curves.

Maximally supersymmetric backgrounds were classified in [7] up to local isometry (see [8] for a purely Lie theoretic proof in the spirit of this note): one has Minkowski spacetime, the Freund-Rubin backgrounds $A d_{4} \times S^{7}$ and $A d_{7} \times S^{4}$, and the KowalskiGlikman symmetric pp-wave. A supersymmetry gap result is also known:

Theorem 1 [13] If a background of $D=11$ supergravity has at least 31 Killing spinors, then it is locally isometric to a maximally supersymmetric background.

The proof relies on a careful analysis of the integrability conditions on the curvature of the superconnection that arise from Killing spinor equations, bosonic field equations, Bianchi identities of the Riemann curvature and $d F=0$. The result was further extended to $N=30$ in [14]. At present, the highest number of Killing spinors known for a non-maximally supersymmetric background is $N=26$, for the pp-wave of [17]. Highly supersymmetric pp-waves are known with any even number $18 \leq N \leq 24$ of Killing spinors [10], whereas typically $N=20$ for Gödel backgrounds [15].

Few general structural results were known until recently:

Theorem 2 [5, 6] Let $(M, g, F)$ be a background of $D=11$ supergravity. Then:

1. There exists an associated Lie superalgebra $\mathfrak{\mathfrak { f }}=\mathfrak{f}_{\overline{0}} \oplus \mathfrak{f}_{\overline{1}}$, where $\mathfrak{I}_{\overline{0}}$ is the space of Killing vectors preserving $F$ and $\mathfrak{f}_{\overline{1}}$ the space of Killing spinors;

2. If $(M, g, F)$ is highly supersymmetric then it is locally homogeneous.

The Lie superalgebra $\mathfrak{f}=\mathfrak{f}_{\overline{0}} \oplus \mathfrak{f}_{\overline{1}}$ is called the Killing superalgebra of the background. Its odd part generates an ideal $\left[\mathfrak{f}_{\overline{1}}, \mathfrak{f}_{\overline{1}}\right] \oplus \mathfrak{f}_{\overline{1}}$, which in this paper call the transvection superalgebra, following a similar terminology used in the context of symmetric spaces. Clearly $N=\operatorname{dim}_{\overline{1}}$ and $\operatorname{dim} \mathfrak{f}_{\overline{1}}>16$ precisely in the highly supersymmetric regime. These results paved the way to use Lie algebraic techniques, but unfortunately many homogeneous supergravity backgrounds are not highly supersymmetric. 
Inspired by the superspace presentation of supergravity theories in terms of Tanaka structures [19, 20], the precise algebraic structure of Killing superalgebras has been derived and further structural results obtained:

Theorem 3 [8] Let $(M, g, F)$ be an 11-dimensional Lorentzian spin manifold with a closed 4-form $F \in \Omega^{4}(M)$. If $\operatorname{dim} \mathfrak{f}_{\overline{1}}>16$, then:

1. The bosonic field equations (1) of $D=11$ supergravity are automatically satisfied;

2. The associated Killing superalgebra is (isomorphic to) a filtered subdeformation of the Poincare superalgebra, the same holds for the transvection superalgebra;

3. The background is fully determined, up to local isometry, by the pair $\left(\varphi, S^{\prime}\right)$, where

$$
\varphi=\left.F^{\sharp}\right|_{o} \in \Lambda^{4} V \text { and } S^{\prime}=\left.\mathfrak{f}_{\overline{1}}\right|_{o} \subset S .
$$

In particular if $\varphi=0$ then $(M, g)$ is locally isometric to Minkowski spacetime.

We call the pair $\operatorname{symb}(M, g, F)=\left(\varphi, S^{\prime}\right)$ given in (3) the geometric symbol of $(M, g, F)$.

This note aims to highlight some aspects and uses of the correspondence between highly supersymmetric supergravity backgrounds and transvection superalgebras.

We will present an alternative and relatively short proof of Theorem 1 , which does not use the restrictions on the holonomy of the superconnection but it is based on the geometric symbol. More precisely, we do not require the Bianchi identities of the Riemann curvature or $d F=0$ (equivalently, the bosonic field equations) but resort to one of the first properties satisfied by the isotropy of the transvection superalgebra of the background, and a partial knowledge of the space of bispinors.

The usage of filtered subdeformations to construct backgrounds has so far been hindered by the amount of algebraic data required to reconstruct the deformation from the symbol. We significantly simplify this data in Theorem 5, paving the way to the generation of new highly supersymmetric backgrounds with prescribed symmetries. The proposed method takes $\operatorname{SO}(V)$-orbits of four-vectors $\varphi \in \Lambda^{4} V$ as starting point and their rank as a useful organizing principle. To date, up to my knowledge, there is no known highly supersymmetric background for which the rank is maximal, i.e., $\operatorname{rk}(\varphi)=11$, and all known backgrounds with $\operatorname{rk}(\varphi)=10$ have $N \leq 22$. On the other hand, those with $N \geq 24$ have rank relatively small: $\operatorname{rk}(\varphi) \leq 8$. Roughly speaking, we might say that the more Killing spinors there are, the more the underlying geometry ought to be rigid and the four-vector less intricate.

The paper has been organized as follows. We first review in $\$ 2$ and $\$ 3$ the basics of filtered subdeformations of the Poincaré superalgebra, including the Reconstruction Theorem [9] and the concepts of Dirac kernel and Lie pair also introduced in [9]. A Lie pair is a pair $\left(\varphi, S^{\prime}\right)$ satisfying a system of coupled algebraic equations, quadratic on $\varphi$ and cubic on $S^{\prime}$, which allows to recover the isotropy algebra of the transvection superalgebra. We then prove Theorem 1 using Dirac kernels and Lie pairs in $\$ 4$,

In $\$ 5$, we turn to study the reconstruction problem for supergravity backgrounds: we prove Theorem 5, introduce the concept of abstract symbol in Definition 3 and state the strong version of the Reconstruction Theorem in Theorem 6.

The last sections $\$ 6$ and $\$ 7$ give details of the strategy to construct supergravity backgrounds in an example, in particular we describe the transvection superalgebras 
of two symmetric pp-waves backgrounds with $N=24$ Killing spinors discovered in [10].

\section{Filtered deformations and the reconstruction theorem}

Let $(V, \eta)$ be the Lorentzian vector space with "mostly minus" signature $(1,10)$ and $S$ the spinor representation of $\mathfrak{s p}(V)$. We recall that $S$ has an $\mathfrak{s p}(V)$-invariant symplectic structure $\langle-,-\rangle$ such that $\left\langle v \cdot s_{1}, s_{2}\right\rangle=-\left\langle s_{1}, v \cdot s_{2}\right\rangle$ for all $s_{1}, s_{2} \in S$ and $v \in V$.

The Poincaré superalgebra $\mathfrak{p}$ has underlying vector space $\mathfrak{s}(V) \oplus S \oplus V$ and nonzero Lie brackets

$$
[A, B]=A B-B A, \quad[A, s]=\sigma(A) s, \quad[A, v]=A v, \quad[s, s]=\kappa(s, s),
$$

where $A, B \in \mathfrak{s p}(V), v \in V$ and $s \in S$. Here $\sigma$ is the spinor representation of $\mathfrak{s o}(V)$ and $\kappa: \odot^{2} S \rightarrow V$ is the so-called Dirac current, defined by

$$
\eta(\kappa(s, s), v)=\langle s, v \cdot s\rangle,
$$

for all $s \in S$ and $v \in V$. One important property of $\kappa$ is that its restriction to $\odot^{2} S^{\prime}$ is surjective on $V$, for any subspace $S^{\prime} \subset S$ with $\operatorname{dim} S^{\prime}>16$ [5]. This algebraic fact is usually referred to as the "local Homogeneity Theorem", due to the role it plays in the proof of Theorem 2 If we grade $p$ so that $\mathfrak{s}(V), S$ and $V$ have degrees $0,-1$ and -2 , respectively, then the above Lie brackets turn $\mathfrak{p}$ into a $\mathbb{Z}$-graded Lie superalgebra

$$
\mathfrak{p}=\mathfrak{p}_{0} \oplus \mathfrak{p}_{-1} \oplus \mathfrak{p}_{-2}, \quad \mathfrak{p}_{0}=\mathfrak{s} \mathfrak{D}(V), \quad \mathfrak{p}_{-1}=S, \quad \mathfrak{p}_{-2}=V .
$$

Note that the parity is consistent with the $\mathbb{Z}$-grading, since $\mathfrak{p}_{\overline{0}}=\mathfrak{p}_{0} \oplus \mathfrak{p}_{-2}$ and $\mathfrak{p}_{\overline{1}}=\mathfrak{p}_{-1}$.

Let $\mathfrak{a}=\mathfrak{h} \oplus S^{\prime} \oplus V$ be a graded subalgebra of $\mathfrak{p}$ with $\operatorname{dim} S^{\prime}>16$ and $\mathfrak{g}$ a filtered deformation of $\mathfrak{a}$, i.e., the Lie brackets of $\mathfrak{g}$ take the following general form [2]:

$$
\begin{aligned}
{[A, B] } & =A B-B A & {[s, s] } & =\kappa(s, s)+\gamma(s, s) \\
{[A, s] } & =\sigma(A) s & {[v, s] } & =\beta(v, s) \\
{[A, v] } & =A v+\delta(A, v) & {[v, w] } & =\alpha(v, w)+\rho(v, w)
\end{aligned}
$$

where $A, B \in \mathfrak{h}, v, w \in V$ and $s \in S^{\prime}$. Here $\alpha \in \operatorname{Hom}\left(\Lambda^{2} V, V\right), \beta \in \operatorname{Hom}\left(V \otimes S^{\prime}, S^{\prime}\right)$, $\gamma \in \operatorname{Hom}\left(\odot^{2} S^{\prime}, \mathfrak{h}\right)$ and $\delta \in \operatorname{Hom}(\mathfrak{h} \otimes V, \mathfrak{h})$ are maps of degree 2 , and $\rho \in \operatorname{Hom}\left(\wedge^{2} V, \mathfrak{h}\right)$ of degree 4. If we do not mention the subalgebra a explicitly, we simply say that $g$ is a highly supersymmetric filtered subdeformation of $\mathfrak{p}$. Finally, we say that $\mathfrak{g}=\mathfrak{g}_{\overline{0}} \oplus \mathfrak{g}_{\overline{1}}$ is odd-generated if $\mathfrak{g}_{\overline{0}}=\left[\mathfrak{g}_{\overline{1}}, \mathfrak{g}_{\overline{1}}\right]$.

The notion of isomorphism $\Phi: \mathfrak{g} \rightarrow \widetilde{\mathfrak{g}}$ of highly supersymmetric filtered subdeformations is given in [9, Def. 5]: it suffices to know that

$$
\Phi(A)=g \cdot A, \quad \Phi(s)=g \cdot s, \quad \text { and } \quad \Phi(v)=g \cdot v+X_{v},
$$


for some $g \in \operatorname{Spin}(V), X: V \rightarrow \widetilde{\mathfrak{h}}$. The notion of embedding is given in [9, Def. 11].

As we now explain, we are interested in filtered deformations that are realizable, that is, corresponding to some highly supersymmetric supergravity background.

Associated to any $\varphi \in \Lambda^{4} V$, there are two natural maps $\beta^{\varphi}: V \otimes S \rightarrow S$ and $\gamma^{\varphi}: \odot^{2} S \rightarrow \mathfrak{s} \mathfrak{0}(V)$, defined by

$$
\begin{aligned}
\beta^{\varphi}(v, s) & =\frac{1}{24}(v \cdot \varphi-3 \varphi \cdot v) \cdot s, \\
\gamma^{\varphi}(s, s) v & =-2 \kappa\left(\beta^{\varphi}(v, s), s\right),
\end{aligned}
$$

for all $s \in S, v \in V$. We will sometimes use also the notation $\beta_{v}^{\varphi}(s)=\beta^{\varphi}(v, s)$. As proven in [8, Proposition 7], these maps are characterized by the "cocyle condition"

$$
\sigma\left(\gamma^{\varphi}(s, s)\right) s=-\beta^{\varphi}(\kappa(s, s), s),
$$

for all $s \in S$, and it turns out that the Spencer cohomology group $H^{2,2}\left(\mathfrak{p}_{-}, \mathfrak{p}\right) \cong \Lambda^{4} V$ precisely via (6)-(7). (In general this cohomology group encodes the Killing spinor equations for supersymmetric field theories, see [8, 3, 4] for details.)

Now, it is well-known that $\odot^{2} S \cong \Lambda^{1} V \oplus \Lambda^{2} V \oplus \Lambda^{5} V$ as an $\mathfrak{s}(V)$-module. This decomposition is unique, since all the three summands are $\mathfrak{s}(V)$-irreducible and inequivalent, so we will consider $\Lambda^{q} V$ directly as a subspace of $\odot^{2} S$, for $q=1,2,5$. We decompose any element $\omega \in \odot^{2} S$ into $\omega=-\frac{1}{32}\left(\omega^{(1)}+\omega^{(2)}+\omega^{(5)}\right)$ accordingly, where $\omega^{(q)} \in \Lambda^{q} V$ for $q=1,2,5$. The overall factor of $-\frac{1}{32}$ is introduced so that $\omega^{(1)}$ coincides exactly with the Dirac current of $\omega$. We may then re-write (7) as:

$$
\begin{aligned}
\eta\left(\gamma^{\varphi}(\omega) v, w\right) & =\frac{1}{6}\left\langle s,\left(2 l_{v} l_{w} \varphi-v \wedge w \wedge \varphi\right) \cdot s\right\rangle \\
& =\frac{1}{3} \eta\left(l_{v} l_{w} \varphi, \omega^{(2)}\right)+\frac{1}{6} \eta\left(l_{v} l_{w} \star \varphi, \omega^{(5)}\right),
\end{aligned}
$$

for all $v, w \in V$, where musical isomorphisms have been tacitly used. (This will also be the case throughout the whole paper, without further mention.) Evidently one has $\operatorname{Ker} \gamma^{\varphi} \supset \Lambda^{1} V$.

Definition 1 [9] A highly supersymmetric filtered subdeformation $g$ of $p$ is realizable if there exists $\varphi \in \Lambda^{4} V$ such that:

1. $\varphi$ is $\mathfrak{h}$-invariant;

2. $\varphi$ is closed, i.e.,

$$
d \varphi\left(v_{0}, \ldots, v_{4}\right)=\sum_{i<j}(-1)^{i+j} \varphi\left(\alpha\left(v_{i}, v_{j}\right), v_{0}, \ldots, \hat{v}_{i}, \ldots, \hat{v}_{j}, \ldots, v_{4}\right)=0
$$

for all $v_{0}, \ldots, v_{4} \in V$;

3. The components of the Lie brackets of $g$ of degree 2 are of the form

$$
\begin{aligned}
\alpha(v, w) & =X_{v} w-X_{w} v & & \beta(v, s)=\beta^{\varphi}(v, s)+\sigma\left(X_{v}\right) s \\
\gamma(s, s) & =\gamma^{\varphi}(s, s)-X_{\kappa(s, s)} & & \delta(A, v)=\left[A, X_{v}\right]-X_{A v}
\end{aligned}
$$

for some linear map $X: V \rightarrow \mathfrak{s o}(V)$, where $A, B \in \mathfrak{h}, v, w \in V$ and $s \in S^{\prime}$. 
It is a non-trivial fact that a realizable $g$ has a unique $\varphi \in \Lambda^{4} V$ that satisfies (1)-(3) of Definition 1 and that the component $\rho$ of degree 4 is fully determined by those of degree 2. (The idea is that $\varphi$ is an element of the cohomology group $H^{2,2}\left(\mathfrak{a}_{-}, \mathfrak{a}\right)$ and, since $H^{4,2}\left(\mathfrak{a}_{-}, \mathfrak{a}\right)=0$, this group fully determines the subdeformation.) Changing the map $X: V \rightarrow \mathfrak{s o}(V)$ by some values in $\mathfrak{h}$ gives isomorphic filtered subdeformations.

For simplicity of exposition, we will call tamed any filtered subdeformation of $p$ that is highly supersymmetric, odd-generated and realizable. We also let

$$
\begin{aligned}
\mathcal{S B} & =\frac{\{\text { highly supersymmetric supergravity bkgds }(M, g, F)\}}{\text { local isometry }} \\
\mathcal{F} \mathcal{D} & =\frac{\{\text { maximal tamed filtered subdeformations } \mathfrak{g} \text { of } \mathfrak{p}\}}{\text { isomorphism }}
\end{aligned}
$$

be the moduli spaces of highly supersymmetric backgrounds and maximal tamed filtered subdeformations. The following result was proved under the assumption that the isotropy group is closed, but it can be easily relaxed (see, e.g., [18]).

Theorem 4 (Reconstruction Theorem.) [9]

1. The assignment $\mathcal{S} B \longrightarrow \mathcal{F} D$ that sends a highly supersymmetric supergravity background to its transvection superalgebra is a $1: 1$ correspondence;

2. The curvature $R: \Lambda^{2} V \rightarrow \mathfrak{s}(V)$ of the supergravity background associated to a $\mathfrak{g} \in \mathcal{F} \mathcal{D}$ is given by $R(v, w)=\rho(v, w)-\left[X_{v}, X_{w}\right]+X_{\alpha(v, w)}$, for all $v, w \in V$.

\section{Dirac kernels and Lie pairs}

Let $\mathfrak{g}$ be a tamed filtered subdeformation of $\mathfrak{p}$, with associated graded Lie algebra $\mathfrak{a}=\mathfrak{h} \oplus S^{\prime} \oplus V$ and $\varphi \in \Lambda^{4} V$. Due to Theorem 4 , we may call $\left(\varphi, S^{\prime}\right)$ the symbol of $\mathfrak{g}$. Since $S^{\prime}$ has dimension $\operatorname{dim} S^{\prime}>16$, then $\odot^{2} S^{\prime} \subset \odot^{2} S$ projects surjectively on $\Lambda^{1} V$ via the Dirac current operation. The embedding $\odot^{2} S^{\prime} \subset \odot^{2} S \cong \Lambda^{1} V \oplus \Lambda^{2} V \oplus \Lambda^{5} V$ is in general diagonal and one cannot expect $\odot^{2} S^{\prime}$ to contain $\Lambda^{q} V$, not even if $q=1$. Restricting the Dirac current to $\odot^{2} S^{\prime}$ gives rise to a short exact sequence

$$
0 \longrightarrow \mathcal{D} \longrightarrow \odot^{2} S^{\prime} \stackrel{\kappa}{\longrightarrow} V \longrightarrow 0,
$$

where

$$
\mathfrak{D}=\odot^{2} S^{\prime} \cap\left(\Lambda^{2} V \oplus \Lambda^{5} V\right)=\left\{\omega \in \odot^{2} S^{\prime} \mid \omega^{(1)}=0\right\}
$$

is called the Dirac kernel of $S^{\prime}$. The Dirac kernel plays a crucial role in our arguments.

A splitting of the above short exact sequence - that is, a linear map $\Sigma: V \rightarrow \odot^{2} S^{\prime}$ such that $\Sigma(v)^{(1)}=v$ for all $v \in V-$ is called a section associated to $S^{\prime}$. A section associated to $S^{\prime}$ always exists and it is unique up to elements in the Dirac kernel.

Definition 2 Let $\mathfrak{h}_{\left(\varphi, S^{\prime}\right)}$ be the subspace of $\mathfrak{s} \mathfrak{D}(V)$ given by 
Remarks on highly supersymmetric backgrounds of 11-dimensional supergravity

$$
\mathfrak{h}_{\left(\varphi, S^{\prime}\right)}=\gamma^{\varphi}(\mathfrak{D})=\left\{\gamma^{\varphi}(\omega) \mid \omega \in \odot^{2} S^{\prime} \text { with } \omega^{(1)}=0\right\} .
$$

The pair $\left(\varphi, S^{\prime}\right)$ is called a Lie pair if

$$
\begin{gathered}
A \cdot \varphi=0 \\
\sigma(A) S^{\prime} \subset S^{\prime}
\end{gathered}
$$

for every $A \in \mathfrak{h}_{\left(\varphi, S^{\prime}\right)}$.

The name "Lie pair" is motivated by the fact that the associated (13) is, in that case, a Lie subalgebra of $\mathfrak{s}(V)$. The following result gives necessary conditions satisfied by any tamed subdeformation.

Proposition 1 [9] Let $\mathrm{g}$ be a tamed filtered subdeformation, with underlying graded Lie algebra $\mathfrak{a}=\mathfrak{h} \oplus S^{\prime} \oplus V$. Then the symbol $\left(\varphi, S^{\prime}\right)$ is a Lie pair and:

1. the isotropy algebra $\mathfrak{h}=\mathfrak{h}_{\left(\varphi, S^{\prime}\right)}$;

2. the map $X: V \rightarrow \mathfrak{s}(V)$ is determined, up to elements in $\mathfrak{h}$, by the identity

$$
X=\gamma^{\varphi} \circ \Sigma,
$$

where $\Sigma$ is any section associated to $S^{\prime}$.

In particular $\mathrm{g}$ is fully determined, up to isomorphism, by the associated symbol.

Corollary 1 Any highly supersymmetric supergravity background $(M, g, F)$ is fully determined by its geometric symbol symb $(M, g, F)$, up to local isometry.

\section{An alternative proof of Theorem 1}

Let $(M, g, F)$ be a background with exactly 31 Killing spinors (locally), and $\left(\varphi, S^{\prime}\right)$ the associated geometric symbol. Since $S^{\prime}$ has dimension 31 , we may write $S^{\prime}=(\mathbb{R} s)^{\perp}$ as the symplectic orthogonal of the line spanned by some spinor $s \in S$ and, as in the original proof of Theorem[1] [13], there are two cases to examine.

Indeed, as shown, e.g., in [1], there are two orbits of $\operatorname{Spin}(V)$ on $\mathbb{P}(S)$, distinguished by the causal character of the associated Dirac current $\xi=\kappa(s, s)$ : either timelike or null. We give our proof of Theorem 1 using Dirac kernels only in the timelike case. Although the overall strategy in the null case is very close, the actual details are different and deserve a separate treatment. We leave them to the interested reader.

We first recall some known facts on realizations of the spinor module $S$ [11, 12] (see also e.g. [9. Proposition 20], which follows the conventions used in this paper). We normalise $s \in S$ so that $\eta(\xi, \xi)=1$ and consider the orthogonal decomposition $V=\mathbb{R} \xi \oplus W$. We set $\Omega^{(q)}=\omega^{(q)}(s, s) \in \Lambda^{q} V$ for $q=1,2,5$; by definition $\Omega^{(1)}=\xi$. The endomorphism $J=\Omega^{(2)} \in \Lambda^{2} V \cong \mathfrak{s} \mathfrak{D}(V)$ acts trivially on $\xi$ and as a complex structure on $W$. Clearly the stabilizer 


$$
\begin{aligned}
\mathfrak{s t a b}_{\mathfrak{s p}(V)}(s) & =\mathfrak{s t a b}_{\mathfrak{s p}(V)}\left(\Omega^{(1)}\right) \cap \mathfrak{s t a b}_{\mathfrak{s o}(V)}\left(\Omega^{(2)}\right) \cap \mathfrak{s t a b}_{\mathfrak{s}(V)}\left(\Omega^{(5)}\right) \\
& =\mathfrak{u}(W, J) \cap \mathfrak{s t a b}_{\mathfrak{s p}(V)}\left(\Omega^{(5)}\right),
\end{aligned}
$$

and it turns out that $\Omega=\Omega^{(5)}+\frac{1}{2} \Omega^{(1)} \wedge \Omega^{(2)} \wedge \Omega^{(2)}$ is the real component of a complex volume form $\Omega-i\left(l_{\xi} \star \Omega\right)$ on $W$. Ultimately $\mathfrak{s t a b}_{\mathfrak{s p}(V)}(s)=\mathfrak{s u}(W, J) \cong \mathfrak{s u}(5)$.

For our purposes, it is sufficient to work at the complexified level. In particular $\mathbb{W}=W \otimes \mathbb{C}$ decomposes as the direct sum of isotropic subspaces $\mathbb{W}=W^{10} \oplus W^{01}$, where $W^{10}=\left\langle w^{10}=\frac{1}{2}(w-i J w) \mid w \in W\right\rangle$ and $W^{01}=\overline{W^{10}}$ are irreducible complex $\mathfrak{s u}(5)$-modules. The Dirac spinor module $\mathbb{S}=S \otimes \mathbb{C}$ is then given by

$$
\mathbb{S}=\Lambda^{(\bullet, 0)}=\bigoplus_{0 \leq p \leq 5} \Lambda_{[5-2 p]}^{(p, 0)},
$$

where $\Lambda^{(p, 0)}=\Lambda^{p}\left(W^{10}\right)^{*}$ is the irreducible $\mathfrak{s u}(5)$-module of the $(p, 0)$-forms on $\mathbb{W}$ and the square brackets denote the charges of $\sigma(2 J)$, with imaginary units removed.

As an aside, we recall that the modules $\Lambda^{(p, q)}$ of $(p, q)$-forms are $\mathfrak{s u}(5)$-irreducible only for $p=0$ or $q=0$ and that for all $0 \leq p, q \leq 5$ we have natural isomorphisms

$$
\Lambda^{(p, 0)} \cong \Lambda^{(0,5-p)} \quad \text { and } \quad \Lambda^{(p, q)} \cong \Lambda^{(5-q, 5-p)} .
$$

For example $\Lambda^{(1,1)} \cong \Lambda_{o}^{(1,1)} \oplus \Lambda^{(0,0)}$ and $\Lambda^{(4,3)} \cong \Lambda^{(2,1)} \cong \Lambda_{o}^{(2,1)} \oplus \Lambda^{(1,0)}$ into irreducible $\mathfrak{s u}(5)$-modules. For any $u \in W^{10}$ and $t \in \mathbb{S}$, the identities

$$
\begin{aligned}
& u \cdot t:=-\sqrt{2} u_{u} t, \\
& \bar{u} \cdot t:=\sqrt{2} \bar{u}^{b} \wedge t, \\
& \xi \cdot t:=\operatorname{vol}_{W} \cdot t,
\end{aligned}
$$

give an irreducible representation of the complex Clifford algebra $\mathbb{C l}(11) \cong 2 \mathbb{C}(32)$, where $\operatorname{vol}_{W} \in \Lambda^{10} W$ is the volume element of $W$. The only trivial $\mathfrak{s u}(5)$-submodules of $\mathbb{S}$ are $\Lambda_{[5]}^{(0,0)}$ and $\Lambda_{[-5]}^{(5,0)}$, more precisely we have

$$
\Lambda_{[-5]}^{(5,0)}=\left\langle s^{(5,0)}=\frac{1}{2}(s+i \xi \cdot s)\right\rangle \quad \text { and } \quad \Lambda_{[5]}^{(0,0)}=\left\langle s^{(0,5)}=\frac{1}{2}(s-i \xi \cdot s)\right\rangle
$$

whence the two elements $s=s^{(5,0)}+s^{(0,5)}$ and $\xi \cdot s=-i\left(s^{(5,0)}-s^{(0,5)}\right)$ do not have a definite charge. The proof of the following lemma is straightforward.

Lemma 1 The space of bispinors $\odot^{2} \mathbb{S}$ decomposes into $\mathfrak{s}(5)$-modules as

$$
\begin{aligned}
\odot^{2} \mathbb{S} & \cong \Lambda^{1} \mathbb{V} \oplus \Lambda^{2} \mathbb{V} \oplus \Lambda^{5} \mathbb{V} \\
& \cong\left(\Lambda^{1} \mathbb{W} \oplus \Lambda^{0} \mathbb{W}\right) \oplus\left(\Lambda^{2} \mathbb{W} \oplus \Lambda^{1} \mathbb{W}\right) \oplus\left(\Lambda^{5} \mathbb{W} \oplus \Lambda^{4} \mathbb{W}\right)
\end{aligned}
$$

with 


$$
\begin{aligned}
\Lambda^{0} \mathbb{W}= & \mathbb{C} \Omega^{(1)} \\
\Lambda^{1} \mathbb{W}= & \Lambda_{[-2]}^{(1,0)} \oplus \Lambda_{[2]}^{(0,1)} \\
\Lambda^{2} \mathbb{W}= & \Lambda_{[-4]}^{(2,0)} \oplus \Lambda_{[4]}^{(0,2)} \oplus \Lambda_{o[0]}^{(1,1)} \oplus \mathbb{C} \Omega^{(2)} \\
\Lambda^{4} \mathbb{W}= & \Lambda_{[-8]}^{(4,0)} \oplus \Lambda_{[8]}^{(0,4)} \oplus \Lambda_{o[-4]}^{(3,1)} \oplus \Lambda_{o[4]}^{(1,3)} \oplus \Lambda_{[-4]}^{(2,0)} \oplus \Lambda_{[4]}^{(0,2)} \\
& \oplus \Lambda_{o[0]}^{(2,2)} \oplus \Lambda_{o[0]}^{(1,1)} \oplus \mathbb{C}\left(\Omega^{(1)} \wedge \Omega^{(2)} \wedge \Omega^{(2)}\right) \\
\Lambda^{5} \mathbb{W}= & \mathbb{C} \Omega \oplus \mathbb{C}\left(l_{\xi} \star \Omega\right) \oplus \Lambda_{o[-6]}^{(4,1)} \oplus \Lambda_{o[6]}^{(1,4)} \oplus \Lambda_{[-6]}^{(3,0)} \oplus \Lambda_{[6]}^{(0,3)} \\
& \oplus \Lambda_{o[-2]}^{(3,2)} \oplus \Lambda_{o[2]}^{(2,3)} \oplus \Lambda_{o[-2]}^{(2,1)} \oplus \Lambda_{o[2]}^{(1,2)} \oplus \Lambda_{[-2]}^{(1,0)} \oplus \Lambda_{[2]}^{(0,1)}
\end{aligned}
$$

into $\mathfrak{s u ( 5 ) - i r r e d u c i b l e s . ~ T h e ~ m o d u l e s ~ n o t ~ i s o m o r p h i c ~ t o ~} \Lambda^{(p, 0)}$ for some $0 \leq p \leq 5$ are colored red. The trivial $\mathfrak{s u}(5)$-modules generated by $\Omega^{(1)}, \Omega^{(2)}$ and $\Omega^{(1)} \wedge \Omega^{(2)} \wedge \Omega^{(2)}$ have charge zero, whereas $\Omega$ together with $\iota_{\xi} \star \Omega$ generate the sum $\Lambda_{[-10]}^{(5,0)} \oplus \Lambda_{[10]}^{(0,5)}$ of the trivial $\mathfrak{s u ( 5 ) - m o d u l e s ~ w i t h ~ c h a r g e ~} \pm 10$.

In particular, the isotypic decomposition of $\odot^{2} \mathbb{S}$ is

$$
\begin{aligned}
\odot^{2} \mathbb{S} \cong & 5 \Lambda^{(0,0)} \oplus 4\left(\Lambda^{(1,0)} \oplus \Lambda^{(0,1)}\right) \oplus 3\left(\Lambda^{(2,0)} \oplus \Lambda^{(0,2)}\right) \oplus 2 \Lambda_{o}^{(1,1)} \oplus \Lambda_{o}^{(2,2)} \\
& \oplus\left(\Lambda_{o}^{(3,1)} \oplus \Lambda_{o}^{(1,3)}\right) \oplus\left(\Lambda_{o}^{(4,1)} \oplus \Lambda_{o}^{(1,4)}\right) \oplus\left(\Lambda_{o}^{(3,2)} \oplus \Lambda_{o}^{(2,3)}\right) \oplus\left(\Lambda_{o}^{(2,1)} \oplus \Lambda_{o}^{(1,2)}\right),
\end{aligned}
$$

where we have grouped conjugated modules with brackets.

Note that $\mathbb{S}=\langle s, \xi \cdot s\rangle \oplus \widetilde{\mathbb{S}}$ decomposes as the direct sum of orthogonal $\mathfrak{s u}(5)$ modules

$$
\langle s, \xi \cdot s\rangle=\Lambda_{[-5]}^{(5,0)} \oplus \Lambda_{[5]}^{(0,0)} \quad \text { and } \quad \widetilde{\mathbb{S}}=\Lambda_{[-3]}^{(4,0)} \oplus \Lambda_{[-1]}^{(3,0)} \oplus \Lambda_{[1]}^{(2,0)} \oplus \Lambda_{[3]}^{(1,0)} .
$$

We would like to estimate the size of the Dirac kernel of $\mathbb{S}^{\prime}=(\mathbb{C} s)^{\perp}=\mathbb{C} s \oplus \widetilde{\mathbb{S}}$ :

Proposition 2 The Dirac kernel $\mathfrak{D}$ of $\mathbb{S}^{\prime}$ includes at least the following elements:

1. Any $\omega \in \Lambda^{(2,0)} \oplus \Lambda^{(0,2)} \subset \Lambda^{2} \mathbb{W}$, modulo $\Lambda^{4} \mathbb{W} \subset \Lambda^{5} \mathbb{V}$;

2. Any $\omega \in \Lambda^{(4,1)} \oplus \Lambda^{(1,4)} \subset \Lambda^{5} \mathbb{W}$, modulo $\Lambda^{2} \mathbb{W} \oplus \Lambda^{4} \mathbb{W} \subset \Lambda^{2} \mathbb{V} \oplus \Lambda^{5} \mathbb{V}$;

3. Any $\omega \in \Lambda^{(3,2)} \oplus \Lambda^{(2,3)} \subset \Lambda^{5} \mathbb{W}$, modulo $\Lambda^{4} \mathbb{W} \subset \Lambda^{5} \mathbb{V}$;

Proof First of all, we note that

$$
\begin{aligned}
\odot^{2} \mathbb{S} & \cong \odot^{2} \mathbb{S}^{\prime} \oplus\left(\mathbb{S}^{\prime} \odot(\xi \cdot s)\right) \oplus \mathbb{C}(\xi \cdot s \odot \xi \cdot s) \\
\odot^{2} \mathbb{S}^{\prime} & \cong \odot^{2} \widetilde{\mathbb{S}} \oplus(\widetilde{\mathbb{S}} \odot s) \oplus \mathbb{C}(s \odot s)
\end{aligned}
$$

into $\mathfrak{s u ( 5 ) - m o d u l e s , ~ a n d ~ a ~ r o u t i n e ~ c o m p u t a t i o n ~ u s i n g ~ ( 2 0 ) ~ g i v e s ~}$ 


$$
\begin{aligned}
\odot^{2} \widetilde{\mathbb{S}} & \cong 2 \Lambda_{[0]}^{(0,0)} \oplus 2\left(\Lambda_{[-2]}^{(1,0)} \oplus \Lambda_{[2]}^{(0,1)}\right) \oplus\left(\Lambda_{[-4]}^{(2,0)} \oplus \Lambda_{[4]}^{(0,2)}\right) \oplus 2 \Lambda_{o[0]}^{(1,1)} \oplus \Lambda_{o[0]}^{(2,2)} \\
& \oplus\left(\Lambda_{o[-4]}^{(3,1)} \oplus \Lambda_{o[4]}^{(1,3)}\right) \oplus\left(\Lambda_{o[-6]}^{(4,1)} \oplus \Lambda_{o[6]}^{(1,4)}\right) \oplus\left(\Lambda_{o[-2]}^{(3,2)} \oplus \Lambda_{o[2]}^{(2,3)}\right) \\
& \oplus\left(\Lambda_{o[-2]}^{(2,1)} \oplus \Lambda_{o[2]}^{(1,2)}\right)
\end{aligned}
$$

and $\widetilde{\mathbb{S}} \odot s \cong \widetilde{\mathbb{S}} \cong\left(\Lambda^{(1,0)} \oplus \Lambda^{(0,1)}\right) \oplus\left(\Lambda^{(2,0)} \oplus \Lambda^{(0,2)}\right)$ into $\mathfrak{s u}(5)$-irreducible modules. The latter ones do not have a definite charge, since $s=s^{(5,0)}+s^{(0,5)} \in \Lambda_{[-5]}^{(5,0)} \oplus \Lambda_{[5]}^{(0,0)}$.

Comparing (21) with the isotypic decomposition of $\odot^{2} \mathbb{S}$ says that all the $\mathfrak{s u}(5)$ submodules colored in red in (19) are contained in $\odot^{2} \widetilde{\mathbb{S}}$. Clearly they also lie in $\mathfrak{D}$. It remains to deal with the isotypic components of type $\Lambda^{(p, 0)}$, where $0 \leq p \leq 4$.

Claim 1 Note that the two copies of $\left(\Lambda^{(2,0)} \oplus \Lambda^{(0,2)}\right)$ with charge \pm 4 are contained in $\Lambda^{2} \mathbb{W}$ and $\Lambda^{4} \mathbb{W}$. Taking an appropriate $t=t^{(4,0)}+t^{(3,0)} \in \Lambda_{[-3]}^{(4,0)} \oplus \Lambda_{[-1]}^{(3,0)} \subset \widetilde{\mathbb{S}}$ and $u_{1}, u_{2} \in W^{10}$, we may use the first equation in (17) to get

$$
\left\langle t,\left(u_{1} \wedge u_{2}\right) \cdot t\right\rangle=2\langle t^{(4,0)}, \underbrace{\left(u_{1} \wedge u_{2}\right) \cdot t^{(3,0)}}_{\text {element of } \Lambda_{[3]}^{(1,0)}}\rangle \neq 0 .
$$

It follows that $\odot^{2} \widetilde{\mathbb{S}}$ projects surjectively to $\Lambda^{(2,0)} \subset \Lambda^{2} \mathbb{W}$ modulo elements in $\Lambda^{4} \mathbb{W}$, whence to $\Lambda^{(2,0)} \oplus \Lambda^{(0,2)}$ as well (because $\widetilde{\mathbb{S}}$ is a module of real type).

$\underline{\text { Claim } 2}$ We already established that $\Lambda_{o[-6]}^{(4,1)} \oplus \Lambda_{o[6]}^{(1,4)} \subset \mathcal{D}$. Now $\Lambda_{[-6]}^{(3,0)} \oplus \Lambda_{[6]}^{(0,3)}$ is the only module of $\odot^{2} \mathbb{S}$ isomorphic to $\Lambda^{(2,0)} \oplus \Lambda^{(0,2)}$ and with charge different from \pm 4 . It follows that $\widetilde{\mathbb{S}} \odot s$ projects surjectively to it, modulo elements in $\Lambda^{2} \mathbb{W} \oplus \Lambda^{4} \mathbb{W}$.

$\underline{\text { Claim } 3}$ We already established that the submodules $\Lambda_{o[-2]}^{(3,2)} \oplus \Lambda_{o[2]}^{(2,3)} \oplus \Lambda_{o[-2]}^{(2,1)} \oplus \Lambda_{o[2]}^{(1,2)}$ of $\Lambda^{5} \mathbb{W}$ belong to $\mathfrak{D}$, so it remains to consider $\Lambda_{[-2]}^{(1,0)} \oplus \Lambda_{[2]}^{(0,1)} \subset \Lambda^{5} \mathbb{W}$.

There are four modules isomorphic to $\Lambda^{(1,0)}$ in $\odot^{2} \mathbb{S}$ : two copies of $\Lambda_{[-2]}^{(1,0)} \subset \odot^{2} \widetilde{\mathbb{S}}$ and two other two copies

$$
\begin{gathered}
s \odot \Lambda_{[3]}^{(1,0)}=\left\{s^{(5,0)} \odot \alpha+s^{(0,5)} \odot \alpha \mid \alpha \in \Lambda_{[3]}^{(1,0)}\right\} \subset s \odot \widetilde{\mathbb{S}}, \\
(\xi \cdot s) \odot \Lambda_{[3]}^{(1,0)}=\left\{s^{(5,0)} \odot \alpha-s^{(0,5)} \odot \alpha \mid \alpha \in \Lambda_{[3]}^{(1,0)}\right\} \subset(\xi \cdot s) \odot \widetilde{\mathbb{S}}
\end{gathered}
$$

that do not have a definite charge. It follows that $2 \Lambda_{[-2]}^{(1,0)} \oplus\left(s \odot \Lambda_{[3]}^{(1,0)}\right) \subset \odot^{2} \mathbb{S}^{\prime}$ projects surjectively to $3 \Lambda_{[-2]}^{(1,0)} \subset \odot^{2} \mathbb{S}$, modulo elements of charge 8. A similar property holds for $3 \Lambda_{[2]}^{(0,1)}$ and this readily implies our last claim.

Let $\left(\varphi, S^{\prime}\right)$ be the geometric symbol of a background with exactly 31 Killing spinors. By Proposition 1 the isotropy $\mathfrak{h}=\mathfrak{h}_{\left(\varphi, S^{\prime}\right)}=\gamma^{\varphi}(\mathfrak{D})$ and the symbol $\left(\varphi, S^{\prime}\right)$ is a Lie pair, in particular $\mathfrak{h} \subset \mathfrak{s t a b}_{\mathfrak{s p}(V)}\left(S^{\prime}\right)=\mathfrak{s t a b}_{\mathfrak{s v}(V)}(\mathbb{R} s)^{\perp}=\mathfrak{s t a b}_{\mathfrak{s p}(V)}(\mathbb{R} s)$. Since $\xi$ is timelike, we actually have 


$$
\mathfrak{h} \subset \mathfrak{s t a b} \mathfrak{b}_{\mathfrak{s p}(V)}(s)=\mathfrak{s u}(5) \subset \mathfrak{s t a b} \mathfrak{b}_{\mathfrak{s}(V)}(\xi) .
$$

The idea is very simple: we write $\varphi=\xi \wedge \phi+\Phi$, for some $\phi \in \Lambda^{3} W$ and $\Phi \in \Lambda^{4} W$ and use the definition (9) of the map $\gamma^{\varphi}$ with $w=\xi$ and $\omega \in \mathfrak{D}$ :

$$
\begin{aligned}
0=\eta\left(\gamma^{\varphi}(\omega) \nu, \xi\right) & =\frac{1}{3} \eta\left(l_{v} l_{\xi} \varphi, \omega^{(2)}\right)+\frac{1}{6} \eta\left(\left(_{\left.l_{v} l_{\xi} \star \varphi, \omega^{(5)}\right)}\right.\right. \\
& =\frac{1}{3} \eta(\underbrace{l_{v} \phi}, \omega^{(2)})+\frac{1}{6} \eta(\underbrace{l_{v} \star \Phi}, \omega^{(5)}),
\end{aligned}
$$

for all $v \in W$, with $\star$ the Hodge star on $W$. We now exploit that $\mathfrak{D}$ is sufficiently big.

Taking $\omega$ as in (1) of Proposition 2, equation (22) becomes $\eta\left(l_{v} \phi, \omega^{(2)}\right)=0$ for all $\omega^{(2)} \in \Lambda^{(2,0)} \oplus \Lambda^{(0,2)} \subset \Lambda^{2} \mathbb{W}$. Decomposing $\phi=\phi^{(3,0)}+\phi^{(2,1)}+\phi^{(1,2)}+\phi^{(0,3)}$ into types and taking $v \in W^{10}$ gives $\phi^{(3,0)}=\phi^{(1,2)}=0$. Similarly $\phi^{(2,1)}=\phi^{(0,3)}=0$, so $\phi=0$. If $\omega$ is now as in (2) of Proposition 2, then $\eta\left(l_{v} \star \Phi, \omega^{(5)}\right)=0$ for all $\omega^{(5)} \in \Lambda^{(4,1)} \oplus \Lambda^{(1,4)}$. Decomposing $\star \Phi \in \Lambda^{6} W$ into types yields $(\star \Phi)^{(p, q)}=0$ except for $(\star \Phi)^{(3,3)}$, but this is zero by (3) of Proposition 2 and a similar argument.

Hence $\varphi=0$, so $(M, g)$ is locally isometric to Minkowski spacetime, in particular it is maximally supersymmetric, a contradiction. The proof is completed.

\section{The reconstruction problem for supergravity backgrounds}

It is not true that every Lie pair has a corresponding filtered subdeformation, in other words, it is the geometric symbol of a background. Indeed, the Lie brackets of a tamed filtered subdeformation $\mathfrak{g}$ are given by

$$
\begin{aligned}
{[A, B] } & =A B-B A & {[s, s] } & =\kappa(s, s)+\gamma^{\varphi}(s, s)-X_{\kappa(s, s)} \\
{[A, s] } & =\sigma(A) s & {[v, s] } & =\beta^{\varphi}(v, s)+\sigma\left(X_{v}\right) s \\
{[A, v] } & =A v+\left[A, X_{v}\right]-X_{A v} & {[v, w] } & =X_{v} w-X_{w} v+\left[X_{v}, X_{w}\right]-X_{X_{v} w-X_{w} v}+R(v, w)
\end{aligned}
$$

for all $A, B \in \mathfrak{h}, v, w \in V, s \in S^{\prime}$. Here $\mathfrak{h}=\mathfrak{h}_{\left(\varphi, S^{\prime}\right)}$ and $X: V \rightarrow \mathfrak{s v}(V)$ is as in (15). The rest of the data $R: \Lambda^{2} V \rightarrow \mathfrak{s o}(V)$ also depends on the Lie pair, as we now recall.

First of all, it bears reminding that the right-hand sides of the above Lie brackets take values in $\mathfrak{a}=\mathfrak{h} \oplus S^{\prime} \oplus V$, but the individual terms may not. Explicitly,

$$
\begin{aligned}
\beta^{\varphi}(v, s)+\sigma\left(X_{v}\right) s & \in S^{\prime} \\
{\left[A, X_{v}\right]-X_{A v} } & \in \mathfrak{h} \\
\gamma^{\varphi}(s, s)-X_{\kappa(s, s)} & \in \mathfrak{h} \\
{\left[X_{v}, X_{w}\right]-X_{X_{v} w-X_{w} v}+R(v, w) } & \in \mathfrak{h}
\end{aligned}
$$

for all $A \in \mathfrak{h}, v, w \in V, s \in S^{\prime}$. Moreover the Lie brackets are subject to the Jacobi identities. There are ten components of them, and five are simply satisfied by 
equivariance and the fact that $\mathfrak{h} \subset \mathfrak{s t a b}_{\mathfrak{s p}(V)}\left(S^{\prime}\right) \cap \mathfrak{s t a b}_{\mathfrak{s p}(V)}(\varphi)[9$, §4.1]. The Jacobi identity with three odd elements is the cocycle condition (8).

The remaining four components of the Jacobi identities are more involved:

- The $[\mathfrak{h} V V]$ Jacobi identity is satisfied if and only if

$$
R: \Lambda^{2} V \rightarrow \mathfrak{s o}(V) \text { is h-equivariant; }
$$

- The $\left[S^{\prime} S^{\prime} V\right]$ Jacobi is equivalent to

$$
\begin{aligned}
\frac{1}{2} R(v, \kappa(s, s)) w & =\kappa\left(\left(X_{v} \beta^{\varphi}\right)(w, s), s\right)+\gamma^{\varphi}\left(\beta_{v}^{\varphi}(s), s\right) w \\
& =\kappa\left(\left(X_{v} \beta^{\varphi}\right)(w, s), s\right)-\kappa\left(\beta_{v}^{\varphi}(s), \beta_{w}^{\varphi}(s)\right)-\kappa\left(\beta_{w}^{\varphi} \beta_{v}^{\varphi}(s), s\right)
\end{aligned}
$$

for all $s \in S^{\prime}, v \in V$ and $w \in V$;

- The $\left[S^{\prime} V V\right]$ Jacobi identity expands to the following condition

$$
R(v, w) s=\left(X_{v} \beta^{\varphi}\right)(w, s)-\left(X_{w} \beta^{\varphi}\right)(v, s)+\left[\beta_{v}^{\varphi}, \beta_{w}^{\varphi}\right](s),
$$

for all $s \in S^{\prime}$ and $v, w \in V$

- Finally, the $[V V V]$ Jacobi identity expands to the algebraic and differential Bianchi identities

$$
\begin{aligned}
R(u, v) w+R(v, w) u+R(w, u) v & =0, \\
\left(X_{u} R\right)(v, w)+\left(X_{v} R\right)(w, u)+\left(X_{w} R\right)(u, v) & =0,
\end{aligned}
$$

for all $u, v, w \in V$.

Using the local Homogeneity Theorem, it is not difficult to see that each of equations (28)-(29) determines uniquely the curvature tensor in terms of the Lie pair.

In summary, if we aim to construct backgrounds via filtered subdeformations and Theorem 4 a long task awaits us: find a Lie pair $\left(\varphi, S^{\prime}\right)$, compute the isotropy $\mathfrak{h}$ and the map $X$ via the Dirac kernel, check that equations (23)-(31) are satisfied, for a putative curvature tensor to be determined, and don't forget also $d \varphi=0$.

The following result drastically simplifies the situation:

Theorem 5 Assume $\left(\varphi, S^{\prime}\right)$ is a Lie pair satisfying (23) and there is $R: \Lambda^{2} V \rightarrow \mathfrak{s o}(V)$ so that (28) and (29) hold. Then the identities (24)-(27) and (30)-(31) automatically hold.

Proof We split the proof in six steps, one for each identity.

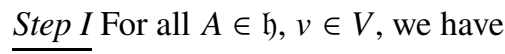

$$
\left[A, X_{v}\right]-X_{A v}=\left[A, \gamma^{\varphi}(\Sigma v)\right]-\gamma^{\varphi}(\Sigma A v)=\gamma^{\varphi}(A \Sigma v-\Sigma A v)
$$

and $A \Sigma v-\Sigma A v \in \mathcal{D}$, since $(A \Sigma v)^{(1)}=A(\Sigma v)^{(1)}=A v=(\Sigma A v)^{(1)}$ and $\Sigma: V \rightarrow \odot^{2} S^{\prime}$. Identity (24) follows then directly from $\mathfrak{h}=\mathfrak{h}_{\left(\varphi, S^{\prime}\right)}=\gamma^{\varphi}(\mathfrak{D})$.

Step II In a similar way $\gamma^{\varphi}(\omega)-X_{\omega^{(1)}}=\gamma^{\varphi}\left(\omega-\Sigma \omega^{(1)}\right)$ is an element of $\mathfrak{h}$ for all $\overline{\omega \in \odot}{ }^{2} S^{\prime}$, proving 25 . 
Step III We now establish (26). Using (28) and the definition of $\gamma^{\varphi}$ we compute

$$
\begin{aligned}
R(v, \kappa(s, s)) w= & 2 \kappa\left(\left(X_{v} \beta^{\varphi}\right)(w, s), s\right)+2 \gamma^{\varphi}\left(\beta_{v}^{\varphi}(s), s\right) w \\
= & 2 \kappa\left(\sigma\left(X_{v}\right)\left(\beta^{\varphi}(w, s)\right), s\right)+\gamma^{\varphi}(s, s) X_{v} w-2 \kappa\left(\beta^{\varphi}\left(w, \sigma\left(X_{v}\right) s\right), s\right) \\
& +2 \gamma^{\varphi}\left(\beta_{v}^{\varphi}(s), s\right) w \\
= & -X_{v}\left(\gamma^{\varphi}(s, s) w\right)-2 \kappa\left(\beta^{\varphi}(w, s), \sigma\left(X_{v}\right) s\right)-2 \kappa\left(\beta^{\varphi}\left(w, \sigma\left(X_{v}\right) s\right), s\right) \\
& +\gamma^{\varphi}(s, s) X_{v} w+2 \gamma^{\varphi}\left(\beta_{v}^{\varphi}(s), s\right) w \\
= & -X_{v}\left(\gamma^{\varphi}(s, s) w\right)+2 \gamma^{\varphi}\left(s, \sigma\left(X_{v}\right) s\right) w+\gamma^{\varphi}(s, s) X_{v} w \\
& +2 \gamma^{\varphi}\left(\beta_{v}^{\varphi}(s), s\right) w \\
= & {\left[\gamma^{\varphi}(s, s), X_{v}\right] w+2 \gamma^{\varphi}(s, \underbrace{\left(\beta_{v}^{\varphi}(s)+\sigma\left(X_{v}\right) s\right)}_{\text {element of } S^{\prime}(\text { by [23) }}) }
\end{aligned}
$$

for all $s \in S^{\prime}, v, w \in V$. On the other hand

$$
\left[X_{v}, X_{\kappa(s, s)}\right]=\left[X_{v}, \gamma^{\varphi}(\Sigma \kappa(s, s))\right]
$$

and

$$
X_{X_{\kappa(s, s)} v}-X_{X_{v} \kappa(s, s)}=X_{\gamma^{\varphi}(\Sigma \kappa(s, s)) v}-X_{X_{v} \kappa(s, s)}-X_{\gamma^{\varphi}(s, s) v}+X_{\gamma^{\varphi}(s, s) v},
$$

for all $s \in S^{\prime}$ and $v \in V$.

We now sum up the three contributions to the identity (26) and regroup the various terms into the sum of

$$
\left[\gamma^{\varphi}(s, s)-\gamma^{\varphi}(\Sigma \kappa(s, s)), X_{v}\right]+X_{\gamma^{\varphi}}(\Sigma \kappa(s, s)) v-X_{\gamma^{\varphi}}(s, s) v
$$

and

$$
2 \gamma^{\varphi}\left(s, \beta_{v}^{\varphi}(s)+\sigma\left(X_{v}\right) s\right)+X_{\gamma^{\varphi}(s, s) v}-X_{X_{v} \kappa(s, s)},
$$

where $\gamma^{\varphi}(s, s)-\gamma^{\varphi}(\Sigma \kappa(s, s)) \in \mathfrak{h}=\gamma^{\varphi}(\mathfrak{D})$. The term (32) belongs to $\mathfrak{h}$ due to (24), which we established in step I. Finally, the Dirac current

$$
\kappa\left(s, \beta_{v}^{\varphi}(s)+\sigma\left(X_{v}\right) s\right)=-\frac{1}{2} \gamma^{\varphi}(s, s) v+\frac{1}{2} X_{v}(\kappa(s, s)),
$$

so the term (33) is also in $\mathfrak{h}$, thanks to the identity (25) established in step II. Step IV We prove that $R$ is $\mathfrak{h}$-equivariant. A direct computation using (28) yields

$$
\left.\frac{1}{2}(A \cdot R)(v, \kappa(s, s)) w=\kappa\left((A \cdot X)_{v} \beta^{\varphi}\right)(w, s), s\right)=\kappa\left(\beta^{(A \cdot X)_{v} \cdot \varphi}(w, s), s\right)=0,
$$

for all $A \in \mathfrak{h}, s \in S^{\prime}$ and $v, w \in V$. Here we used that $(A \cdot X)_{v}=\left[A, X_{v}\right]-X_{A v}$ is an element of $\mathfrak{h}$ by step $\mathrm{I}$, hence it annihilates $\varphi$.

Step $V$ It is sufficient to establish (30) with $w=\kappa(s, s)$ for all $s \in S^{\prime}$. Now 


$$
\begin{aligned}
\frac{1}{2}(R(v, \kappa(s, s)) u+R(\kappa(s, s), u) v)= & \kappa\left(\left(X_{v} \beta^{\varphi}\right)(u, s), s\right)-\kappa\left(\left(X_{u} \beta^{\varphi}\right)(v, s), s\right) \\
& -\kappa\left(\left[\beta_{u}^{\varphi}, \beta_{v}^{\varphi}\right](s), s\right)
\end{aligned}
$$

by equation (28) and this term is also equal to $\frac{1}{2} R(v, u) \kappa(s, s)=\kappa(R(v, u) s, s)$ by (29).

Step VIThe last step is the most involved and needs a preliminary crucial observation: it is enough to establish (31) when one of the elements of $V$ is of the form $\omega^{(1)}$ for some $\omega \in \odot^{2} S^{\prime}$ in the image of the section $\Sigma: V \rightarrow \odot^{2} S^{\prime}$. For simplicity of exposition, we denote $\omega=s \odot s$ with $s \in S^{\prime}$, although it is really a sum of decomposable bispinors. Our assumption on $\omega$ reads then $s \odot s=\omega=\Sigma \omega^{(1)}=\Sigma(\kappa(s, s))$, whence

$$
X_{\kappa(s, s)}=\gamma^{\varphi}(\Sigma(\kappa(s, s)))=\gamma^{\varphi}(s, s) .
$$

We will crucially use this property in the proof.

Now $A \cdot \beta^{\varphi}=\beta^{A \cdot \varphi}$ for all $A \in \mathfrak{s p}(V)$ by $\mathfrak{s p}(V)$-equivariance. A direct computation using this fact and (28) yields

$$
\begin{aligned}
\frac{1}{2}(A \cdot R)(v, \kappa(s, s)) w= & \kappa\left(\beta^{\left(A \cdot X_{v} \cdot \varphi\right)}(w, s), s\right)-\kappa\left(\beta^{\left(X_{A v} \cdot \varphi\right)}(w, s), s\right) \\
& +\gamma^{A \cdot \varphi}\left(\beta_{v}^{\varphi}(s), s\right) w+\gamma^{\varphi}\left(\beta_{v}^{(A \cdot \varphi)}(s), s\right) w,
\end{aligned}
$$

for all $A \in \mathfrak{s o}(V)$. Applying (35) with $A=X_{u}$ and skew-symmetrizing in $u$ and $v$ says that the contribution

$$
\frac{1}{2}\left(X_{u} \cdot R\right)(v, \kappa(s, s)) w-\frac{1}{2}\left(X_{v} \cdot R\right)(u, \kappa(s, s)) w
$$

to the differential Bianchi identity is given by

$$
\begin{aligned}
\kappa\left(\beta^{\left(\left[X_{u}, X_{v}\right] \cdot \varphi\right)}(w, s), s\right)-\kappa\left(\beta^{\left(X_{X_{u} v-X_{v} u} \cdot \varphi\right)}(w, s), s\right)+\gamma^{\varphi}\left(\beta_{v}^{\left(X_{u} \cdot \varphi\right)}(s), s\right) w \\
-\gamma^{\varphi}\left(\beta_{u}^{\left(X_{v} \cdot \varphi\right)}(s), s\right) w+\gamma^{X_{u} \cdot \varphi}\left(\beta_{v}^{\varphi}(s), s\right) w-\gamma^{X_{v} \cdot \varphi}\left(\beta_{u}^{\varphi}(s), s\right) w .
\end{aligned}
$$

Using identity (26), established in step III, and the definition of a Lie pair, we directly see that the first two terms in (37) are equal to

$$
-\kappa\left(\beta^{(R(u, v) \cdot \varphi)}(w, s), s\right)=\frac{1}{2} \gamma^{(R(u, v) \cdot \varphi)}(s, s) w .
$$

We recall our crucial assumption (34) and turn to compute the last contribution to the differential Bianchi identity:

$$
\begin{aligned}
\frac{1}{2}\left(X_{\kappa(s, s)} \cdot R\right)(u, v)= & \frac{1}{2}\left(\gamma^{\varphi}(s, s) \cdot R\right)(u, v) \\
= & -\frac{1}{2}\left[R(u, v), \gamma^{\varphi}(s, s)\right]-\frac{1}{2} R\left(\gamma^{\varphi}(s, s) u, v\right)-\frac{1}{2} R\left(u, \gamma^{\varphi}(s, s) v\right) \\
= & -\frac{1}{2} \gamma^{(R(u, v) \cdot \varphi)}(s, s)-\gamma^{\varphi}(R(u, v) s, s)-\frac{1}{2} R\left(\gamma^{\varphi}(s, s) u, v\right) \\
& -\frac{1}{2} R\left(u, \gamma^{\varphi}(s, s) v\right),
\end{aligned}
$$


where we used that $A \cdot \gamma^{\varphi}=\gamma^{A \cdot \varphi}$ for all $A \in \mathfrak{s p}(V)$. Thanks to (29) we may expand one of the terms in the next-to-last line:

$$
\begin{aligned}
-\gamma^{\varphi}(R(u, v) s, s)= & -\gamma^{\varphi}\left(\beta^{\left(X_{u} \cdot \varphi\right)}(v, s), s\right)+\gamma^{\varphi}\left(\beta^{\left(X_{v} \cdot \varphi\right)}(u, s), s\right) \\
& -\gamma^{\varphi}\left(\left[\beta_{u}^{\varphi}, \beta_{v}^{\varphi}\right](s), s\right) .
\end{aligned}
$$

Let us collect what we obtained so far: summing up (37) and (39), and using (38) and (40), we are left with

$$
\begin{aligned}
\gamma^{X_{u} \cdot \varphi}\left(\beta_{v}^{\varphi}(s), s\right) w & -\gamma^{X_{v} \cdot \varphi}\left(\beta_{u}^{\varphi}(s), s\right) w-\gamma^{\varphi}\left(\left[\beta_{u}^{\varphi}, \beta_{v}^{\varphi}\right](s), s\right) \\
& -\frac{1}{2} R\left(\gamma^{\varphi}(s, s) u, v\right) w-\frac{1}{2} R\left(u, \gamma^{\varphi}(s, s) v\right) w .
\end{aligned}
$$

Using the definition of $\gamma^{\varphi}$, equation (28) and a direct computation, we see that the second line of 41) is equal to

$$
\begin{aligned}
R\left(\kappa\left(\beta_{u}^{\varphi}(s), s\right), v\right) w-R\left(\kappa\left(\beta_{v}^{\varphi}(s), s\right), u\right) w= & -\gamma^{X_{u} \cdot \varphi}\left(\beta_{v}^{\varphi}(s), s\right) w+\gamma^{X_{v} \cdot \varphi}\left(\beta_{u}^{\varphi}(s), s\right) w \\
& +\gamma^{\varphi}\left(\left[\beta_{u}^{\varphi}, \beta_{v}^{\varphi}\right](s), s\right)
\end{aligned}
$$

so (41) vanishes and the proof is completed.

Motivated by this result, we give the following definition.

Definition 3 Let $\left(\varphi, S^{\prime}\right)$ be a Lie pair, that is, $S^{\prime}$ is a subspace of $S$ with $\operatorname{dim} S^{\prime}>16$,

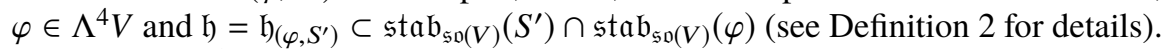
Let $\Sigma: V \rightarrow \odot^{2} S^{\prime}$ be any section associated with $S^{\prime}$ and set $X=\gamma^{\varphi} \circ \Sigma: V \rightarrow \mathfrak{s o}(V)$ and $\alpha(v, w)=X_{v} w-W_{w} v$ for all $v, w \in V$. Then $\left(\varphi, S^{\prime}\right)$ is called an abstract symbol if

$$
\begin{aligned}
\beta^{\varphi}(v, s)+\sigma\left(X_{v}\right) s & \in S^{\prime} \\
d \varphi\left(v_{0}, \ldots, v_{4}\right) & =\sum_{i<j}(-1)^{i+j} \varphi\left(\alpha\left(v_{i}, v_{j}\right), v_{0}, \ldots, \hat{v}_{i}, \ldots, \hat{v}_{j}, \ldots, v_{4}\right)=0 \\
\frac{1}{2} R(v, \kappa(s, s)) w & =\kappa\left(\left(X_{v} \beta^{\varphi}\right)(w, s), s\right)+\gamma^{\varphi}\left(\beta_{v}^{\varphi}(s), s\right) w \\
R(v, w) s & =\left(X_{v} \beta^{\varphi}\right)(w, s)-\left(X_{w} \beta^{\varphi}\right)(v, s)+\left[\beta_{v}^{\varphi}, \beta_{w}^{\varphi}\right](s)
\end{aligned}
$$

for some $R: \Lambda^{2} V \rightarrow \mathfrak{s o}(V)$ and all $v, w, v_{0}, \ldots, v_{4} \in V, s \in S^{\prime}$.

Combining Theorem 4 Corollary 1 and the discussion carried out in this section, we arrive at the following version of the Reconstruction Theorem. Therein

$$
\mathcal{A S}=\frac{\left\{\text { abstract symbols }\left(\varphi, S^{\prime}\right)\right\}}{\operatorname{Spin}(V)}
$$

is the moduli space of abstract symbols.

Theorem 6 (Reconstruction Theorem - Strong Version) The map $\mathcal{S} B \longrightarrow \mathcal{A} S$ that sends a highly supersymmetric supergravity background to its geometric symbol is a $1: 1$ correspondence, with image the space of abstract symbols. 
The construction of supergravity backgrounds breaks then into three steps:

1. Describe $\operatorname{Spin}(V)$-orbits of Lie pairs $\left(\varphi, S^{\prime}\right)$ (and therefore the associated graded subalgebras $\mathfrak{a}=\mathfrak{h}_{\left(\varphi, S^{\prime}\right)} \oplus S^{\prime} \oplus V$ of the Poincaré superalgebra);

2. Check (42) and (43) (roughly speaking, this gives the transvection superalgebra of the background at the level of infinitesimal deformation);

3. See if there exists $R: \Lambda^{2} V \rightarrow \mathfrak{s o}(V)$ satisfying (45) and then check (44) (at this stage we fully reconstructed the transvection superalgebra as a deformation).

Remark 1 I have some evidence that (44) is a consequence of the other identities, but I don't have a complete proof so far.

Remark 2 If $\varphi \in \Lambda^{4} V$ appears in a Lie pair $\left(\varphi, S^{\prime}\right)$ with $S^{\prime}=S$ then $\varphi$ is decomposable, cf. [8]. It would be desirable to have an a priori understanding of which $\varphi \in \Lambda^{4} V$ appear in Lie pairs at all. The variety of such four-vectors is $\mathrm{SO}(V)$-stable and in most likelihood properly contained in $\Lambda^{4} V$.

It is clear that constructing Lie pairs remains the most difficult step, since they are defined by a rather complicated system of coupled algebraic equations, quadratic on $\varphi$ and cubic on $S^{\prime}$. To a certain extent, this can be regarded as the algebraic counterpart of the bosonic field equations (1) for highly supersymmetric backgrounds. We here propose a "separation of variables" technique to settle it:

- For a given $\varphi \in \Lambda^{4} V$, we consider the operator $\gamma^{\varphi}: \odot^{2} S \rightarrow \mathfrak{s} \mathfrak{p}(V)$ and determine the intersection

$$
\widetilde{\mathfrak{h}}=\operatorname{Im} \gamma^{\varphi} \cap \mathfrak{s t a b} \mathfrak{b}_{\mathfrak{s}(V)}(\varphi)
$$

of its full image with the stabilizer of $\varphi$. It is easy to see that this is a Lie subalgebra of $\mathfrak{s p}(V)$. We also determine the $\widetilde{\mathfrak{h}}$-submodule $K=\left(\gamma^{\varphi}\right)^{-1}\left(\mathfrak{s t a b}_{\mathfrak{s p}(V)}(\varphi)\right)$ of $\odot^{2} S$;

- We choose a presentation of $S$ adapted to $\widetilde{\mathfrak{h}}$ and look for $\widetilde{\mathfrak{h}}$-submodules $S^{\prime}$ of $S$. We identify those which satisfy the inclusion $\mathfrak{D} \subset K$ using $\widetilde{\mathfrak{b}}$-equivariance.

Note that the subspace $S^{\prime}$ only enters at the last stage and that the inclusion there is a quadratic relation on spinors, and not cubic. A pair $\left(\varphi, S^{\prime}\right)$ so obtained is a Lie pair. Indeed

$$
\mathfrak{h}=\gamma^{\varphi}(\mathfrak{D}) \subset \gamma^{\varphi}(K) \subset \mathfrak{s t a b} \mathfrak{s p}_{\mathfrak{s}(V)}(\varphi)
$$

and $\mathfrak{h} \subset \mathfrak{s t a b}_{\mathfrak{s} \mathfrak{0}(V)}\left(S^{\prime}\right)$, since $S^{\prime}$ is an $\widetilde{\mathfrak{h}}$-module by construction and $\mathfrak{h} \subset \widetilde{\mathfrak{h}}$ by (46).

In \$7 we work out an example where $V=\mathbb{R}^{1,1} \oplus \mathbb{R}^{9}$ splits into the ortohognal direct sum of $\mathbb{R}^{1,1}=\left\{\boldsymbol{e}_{+}, \boldsymbol{e}_{-}\right\}$and $\mathbb{R}^{9}=\left\{\boldsymbol{e}_{1}, \ldots, \boldsymbol{e}_{9}\right\}$, and $\varphi=\boldsymbol{e}_{+} \wedge \phi$ for some $\phi \in \Lambda^{3} \mathbb{R}^{9}$ of small rank. The relevant orbits are described in $\$ 6$

\section{The $\mathrm{SO}_{9}(\mathbb{R})$-orbits in $\Lambda^{3} \mathbb{R}^{9}$ of subminimal rank}

In this section, we set $G=\mathrm{SL}_{9}(\mathbb{R})$ and let $G^{\theta}=\mathrm{SO}_{9}(\mathbb{R})$ be the special orthogonal subgroup, i.e., the fixed point set of the Cartan involution $\theta: G \rightarrow G$ of $G$. We are interested in the stratification under the action of $G^{\theta}$ of small orbits of $G$ on $\Lambda^{3} \mathbb{R}^{9}$. 
The support of a trivector $\phi \in \Lambda^{3} \mathbb{R}^{9}$ is the unique minimal subspace $\mathbb{E} \subset \mathbb{R}^{9}$ such that $\phi \in \Lambda^{3} \mathbb{E}$. Its dimension is called the rank of $\phi$ and it is a $G$-invariant, in particular it is one of the simplest $G^{\theta}$-invariants, together with the trivector's norm.

The trivectors of minimal (non-zero) rank are decomposable and form a $G$-orbit, which is stratified by the level sets of the norm in a 1-parameter family of $G^{\theta}$-orbits. The associated filtered subdeformations have been studied in [8]:

Proposition 3 Let $\phi=\lambda \boldsymbol{e}_{123}$ for some $\lambda>0$ and $(M, g, F)$ a highly supersymmetric supergravity background with $\operatorname{symb}(M, g, F)=\left(\varphi=\boldsymbol{e}_{+} \wedge \phi, S^{\prime}\right)$ for some $S^{\prime} \subset S$. Then $S^{\prime}=S$ and $(M, g, F)$ is locally isometric to the Kowalski-Glikman background.

Proof The transvection superalgebra of the Kowalski-Glikman background is the filtered subdeformation with symbol $(\varphi, S)[8]$. By maximality, the symbol $\left(\varphi, S^{\prime}\right)$ of the transvection superalgebra of our background has to coincide with $(\varphi, S)$.

The next step in the analysis of filtered deformations and supergravity backgrounds is the rank 5 orbit, due to the following well-known result:

Lemma 2 An indecomposable $\phi \in \Lambda^{3} \mathbb{R}^{9}$ has rank at least 5 . The rank 5 trivectors constitute a $G$-orbit, with the representative, e.g., $\phi=\boldsymbol{e}_{123}+\boldsymbol{e}_{145}$.

This is also a subminimal orbit, in the sense that its Zariski-closure consists of the orbit itself, the minimal orbit of the non-zero decomposable trivectors and the zero trivector, see for instance [16, page 104]. We will therefore denote it by $O_{\text {submin }}^{G}$.

Proposition $4\left(G^{\theta}\right.$-orbits of rank 5 trivectors.) The subminimal orbit $O_{\text {submin }}^{G}$ admits a stratification

$$
O_{\text {submin }}^{G}=\bigcup_{0<\lambda \leq \mu} O_{\phi_{(\lambda, \mu)}}^{G^{\theta}},
$$

where $O_{\phi_{(\lambda, \mu)}}^{G^{\theta}}$ is the $G^{\theta}$-orbit of the trivector $\phi_{(\lambda, \mu)}=\lambda \boldsymbol{e}_{123}+\mu \boldsymbol{e}_{145}$. The Lie algebra of the stabiliser $H^{\theta}$ of $\phi_{(\lambda, \mu)}$ in $G^{\theta}$ is

$$
\mathfrak{h}^{\theta}= \begin{cases}\left\langle\boldsymbol{e}_{23}, \boldsymbol{e}_{45}\right\rangle \oplus \mathfrak{s v}\left(\mathbb{E}^{\perp}\right) \cong \mathfrak{u}(1) \oplus \mathfrak{u}(1) \oplus \mathfrak{s v}\left(\mathbb{E}^{\perp}\right) & \text { if } \lambda<\mu, \\ \left\langle\boldsymbol{e}_{23}, \boldsymbol{e}_{45}, \boldsymbol{e}_{24}+\boldsymbol{e}_{35}, \boldsymbol{e}_{25}-\boldsymbol{e}_{34}\right\rangle \oplus \mathfrak{s v}\left(\mathbb{E}^{\perp}\right) \cong \mathfrak{u}(2) \oplus \mathfrak{s v}\left(\mathbb{E}^{\perp}\right) & \text { if } \lambda=\mu,\end{cases}
$$

where $\mathbb{E}=\left\langle\boldsymbol{e}_{1}, \ldots, \boldsymbol{e}_{5}\right\rangle$ is the support of $\phi_{(\lambda, \mu)}$ and $\mathbb{E}^{\perp}=\left\langle\boldsymbol{e}_{6}, \ldots, \boldsymbol{e}_{9}\right\rangle$.

Proof Let $\phi \in \Lambda^{3} \mathbb{R}^{9}$ be a rank 5 trivector. Then $g \cdot \phi$ has support $\mathbb{E}=\left\langle e_{1}, \ldots, e_{5}\right\rangle$ for some $g \in G^{\theta}$, and two trivectors with support $\mathbb{E}$ are in the same $G^{\theta}$-orbit if and only if they are in the same $\mathrm{O}(\mathbb{E})$-orbit. Therefore, it is enough to describe the $\mathrm{O}(\mathbb{E})$-orbits of trivectors $\phi \in \Lambda^{3} \mathbb{E}$ of rank 5. (We will see that they coincide with $\mathrm{SO}(\mathbb{E})$-orbits.)

We fix a volume element vol $\in \Lambda^{5} \mathbb{E}^{*}$ and consider the bijection

$$
\Lambda^{3} \mathbb{E} \rightarrow \Lambda^{2} \mathbb{E}^{*}, \quad \phi \mapsto l_{\phi} \text { vol },
$$

which is an isomorphism of $\mathrm{SO}(\mathbb{E})$-modules. Now $\Lambda^{2} \mathbb{E}^{*} \cong \mathfrak{s}(\mathbb{E})$, so the $\mathrm{SO}(\mathbb{E})$-orbits on $\Lambda^{3} \mathbb{E}$ are in bijective correspondence with the adjoint orbits. The group $\operatorname{SO}(\mathbb{E})$ is compact, hence any adjoint orbit has a representative in the Cartan subalgebra 


$$
\mathrm{t}=\left\{\left(\begin{array}{c|cc|c}
0 & & & \\
\hline & 0 & \mu & \\
& -\mu & 0 & \\
\hline & & 0 & \lambda \\
& & -\lambda & 0
\end{array}\right) \mid \lambda, \mu \in \mathbb{R}\right\}
$$

of $\mathfrak{s p}(\mathbb{E})$. Using the Weyl group of $\mathrm{SO}(\mathbb{E})$, we may uniquely arrange for $0 \leq \lambda \leq \mu$. The corresponding trivector is $\phi=\lambda \boldsymbol{e}_{123}+\mu \boldsymbol{e}_{145}$ and has rank 5 if and only if $\lambda \neq 0$.

The last claim follows from a direct computation, which we omit.

Remark 3 A similar analysis for higher rank orbits is possible but it is more involved, as the connection with the adjoint orbits of a compact Lie group is not available in general. A possible strategy is outlined here:

1. Let $O_{\phi}^{G} \cong G / H$ be the $G$-orbit with representative $\phi \in \Lambda^{3} \mathbb{R}^{9}$ of rank $6 \leq k \leq 9$, where $H$ is the stabiliser of $\phi$ in $G$. We then have a stratification

$$
O_{\phi}^{G}=\bigcup_{i} O_{\phi_{i}}^{G^{\theta}}
$$

under the action of $G^{\theta}$, which is parametrised by the double cosets in $G^{\theta} \backslash G / H$;

2. The $H$-equivariant map $G^{\theta} g \mapsto g^{t} \cdot g$ identifies $G^{\theta} \backslash G$ with the space $S y m_{9}^{+}$of positive-definite symmetric matrices with unit determinant. (Surjectivity follows from Sylvester's law of inertia, the right action of $\mathrm{H}$ on $\mathrm{Sym}_{9}^{+}$is by congruence.) Hence

$$
G^{\theta} \backslash G / H \cong S y m_{9}^{+} / H
$$

parametrises the stratification (47);

3. Tipically we have a non-trivial Levi decomposition

$$
H=L \ltimes U
$$

of $H$ with reductive subgroup $L$ and unipotent radical $U$. To compute $S y m_{9}^{+} / H$, we may first consider $S y m_{9}^{+} / U$ and then the residual action of $L \cong H / U$ on it.

The orbits $O_{\phi}^{G}$ of trivectors of rank at most 9 have been determined in [21] (upon complexification). The description of those of rank 9 is extremely involved but those of rank $\leq 8$ are automatically nilpotent (in the sense of Vinberg's theory of $\theta$-groups) and relatively few: upon complexification, there are 13 orbits $O_{\phi}^{G}$ of rank 8 and the orbits of rank $\leq 7$ are given by: 


\begin{tabular}{|c|c|c|c|c|c|}
\hline class & representative $\phi$ & rank & reductive part I of $\mathfrak{l}$ & $\operatorname{dim} \mathrm{I}$ & $\operatorname{dim} \mathfrak{h}$ \\
\hline \hline 90 & $\boldsymbol{e}_{123}+\boldsymbol{e}_{147}+\boldsymbol{e}_{257}+\boldsymbol{e}_{367}+\boldsymbol{e}_{456}$ & 7 & $G_{2} \oplus A_{1}$ & 17 & 31 \\
\hline 93 & $\boldsymbol{e}_{125}+\boldsymbol{e}_{137}+\boldsymbol{e}_{247}+\boldsymbol{e}_{346}$ & 7 & $3 A_{1} \oplus \mathbb{C}$ & 10 & 32 \\
\hline 94 & $\boldsymbol{e}_{127}+\boldsymbol{e}_{134}+\boldsymbol{e}_{256}$ & 7 & $3 A_{1} \oplus 2 \mathbb{C}$ & 11 & 35 \\
\hline 95 & $\boldsymbol{e}_{125}+\boldsymbol{e}_{136}+\boldsymbol{e}_{147}+\boldsymbol{e}_{234}$ & 7 & $A_{2} \oplus A_{1} \oplus \mathbb{C}$ & 12 & 38 \\
\hline 96 & $\boldsymbol{e}_{123}+\boldsymbol{e}_{456}$ & 6 & $3 A_{2}$ & 24 & 42 \\
\hline 97 & $\boldsymbol{e}_{124}+\boldsymbol{e}_{135}+\boldsymbol{e}_{236}$ & 6 & $2 A_{2} \oplus \mathbb{C}$ & 17 & 43 \\
\hline 99 & $\boldsymbol{e}_{123}+\boldsymbol{e}_{145}+\boldsymbol{e}_{167}$ & 7 & $C_{3} \oplus A_{1} \oplus \mathbb{C}$ & 25 & 45 \\
\hline 100 & $\boldsymbol{e}_{123}+\boldsymbol{e}_{145}$ & 5 & $A_{3} \oplus C_{2} \oplus \mathbb{C}$ & 26 & 50 \\
\hline 101 & $\boldsymbol{e}_{123}$ & 3 & $A_{5} \oplus A_{2}$ & 43 & 61 \\
\hline
\end{tabular}

where a couple of misprints in [21] have been corrected. Some orbits (but not all) have been investigated in [10] in the context of pp-waves.

By previous steps, the stratification (47) of an orbit $O_{\phi}^{G}$ is parametrised by unit volume scalar products up to $H$-equivalence. The study of the associated supergravity backgrounds will be considered in future work, we now work out our example. We will omit most of the actual details of this calculation.

\section{Example}

The proof of the following lemma is straightforward.

Lemma 3 Let $\varphi \in \Lambda^{4} V$ be of the form $\varphi=\boldsymbol{e}_{+} \wedge \phi$ for some non-zero $\phi \in \Lambda^{3} \mathbb{R}^{9}$. Then

$$
\mathfrak{s t a b}_{\mathfrak{s p}(V)}(\varphi)=\mathfrak{s t a b}_{\mathfrak{s p}\left(\mathbb{R}^{9}\right)}(\phi) \ltimes\left(\boldsymbol{e}_{+} \wedge \mathbb{R}^{9}\right),
$$

in particular $\mathfrak{s t a b}_{\mathfrak{s p}(V)}(\varphi) \subset \mathfrak{s t a b}_{\mathfrak{s p}(V)}\left(\boldsymbol{e}_{+}\right)$.

We consider the case where $\phi$ has rank 5 with maximum stabilizer, i.e., $\phi=\boldsymbol{e}_{123}+$ $\boldsymbol{e}_{145}$, up to positive multiples. It is well known that the field equations (1) are invariant under a homothety that rescales both the metric and the 4-form and that the associated transvection superalgebras are not isomorphic as filtered subdeformations. However, they are isomorphic if we simply allow for $g \in \mathrm{CSpin}(V)$ in equation (5), so we may indeed restrict to $\phi=\boldsymbol{e}_{123}+\boldsymbol{e}_{145}$. We recall that $\mathbb{R}^{9}=\mathbb{E} \oplus \mathbb{E}^{\perp}$, where $\mathbb{E}=\left\langle\boldsymbol{e}_{1}, \ldots, \boldsymbol{e}_{5}\right\rangle$ is the support of $\phi$ and $\mathbb{E}^{\perp}=\left\langle\boldsymbol{e}_{6}, \ldots, \boldsymbol{e}_{9}\right\rangle$.

Now $\mathfrak{s t a b}_{\mathfrak{s}(V)}(\varphi)=\left(\mathfrak{u}(2) \oplus \mathfrak{s} \mathfrak{D}\left(\mathbb{E}^{\perp}\right)\right) \ltimes\left(\boldsymbol{e}_{+} \wedge \mathbb{R}^{9}\right)$ according to Proposition 4 and the image $\operatorname{Im} \gamma^{\varphi}$ is a 36-dimensional subspace of $\mathfrak{s D}(V)$ satisfying

$$
\widetilde{\mathfrak{h}}=\operatorname{Im} \gamma^{\varphi} \cap \mathfrak{s t a b}_{\mathfrak{s} \mathfrak{D}(V)}(\varphi)=\left(\mathfrak{u}(1) \oplus \mathfrak{s \mathfrak { D }}\left(\mathbb{E}^{\perp}\right)\right) \ltimes\left(\boldsymbol{e}_{+} \wedge \mathbb{R}^{9}\right),
$$

with $\mathfrak{u}(1)=\mathbb{R}\left(\boldsymbol{e}_{23}+\boldsymbol{e}_{45}\right)$. The kernel $\operatorname{Ker} \gamma^{\varphi}$ is 492-dimensional.

We use the isomorphism of algebras $C \ell(V) \cong C \ell\left(\mathbb{R}^{9}\right) \otimes C \ell\left(\mathbb{R}^{1,1}\right) \cong 2 \mathbb{R}(16) \otimes \mathbb{R}(2)$ (we recall that $\mathbb{R}^{9}$ is negative definite for us) to write

$$
S \cong \mathbb{R}^{16} \otimes \mathbb{R}^{2} \cong S_{+} \oplus S_{-}
$$


with $S_{ \pm} \cong \mathbb{R}^{16}$ and define the Gamma matrices as block matrices with square blocks of order 16 :

$$
\Gamma_{+}=\sqrt{2}\left(\begin{array}{cc}
0 & \mathrm{Id} \\
0 & 0
\end{array}\right), \quad \Gamma_{-}=-\sqrt{2}\left(\begin{array}{cc}
0 & 0 \\
\mathrm{Id} & 0
\end{array}\right), \quad \Gamma_{i}=\left(\begin{array}{cc}
\gamma_{i} & 0 \\
0 & -\gamma_{i}
\end{array}\right) \quad(i=1, \ldots, 9) .
$$

The Gamma matrices $\gamma_{i} \in C \ell\left(\mathbb{R}^{9}\right)$ will be described via a quaternionic formalism.

First of all $C \ell\left(\mathbb{R}^{5}\right) \cong 2 \mathbb{H}(2)$ and an isomorphism is given by the quaternionic matrices

$$
A_{1}=\left(\begin{array}{cc}
1 & 0 \\
0 & -1
\end{array}\right), A_{2}=\left(\begin{array}{ll}
0 & 1 \\
1 & 0
\end{array}\right), A_{3}=\left(\begin{array}{cc}
0 & L_{i} \\
-L_{i} & 0
\end{array}\right), A_{4}=\left(\begin{array}{cc}
0 & L_{j} \\
-L_{j} & 0
\end{array}\right), A_{5}=\left(\begin{array}{cc}
0 & L_{k} \\
-L_{k} & 0
\end{array}\right),
$$

where $L_{q}: \mathbb{H} \rightarrow \mathbb{H}$ is left multiplication by $q \in \mathbb{H}$. We set $\mathbb{R}^{16} \cong \mathbb{H}^{2} \otimes \mathbb{R}^{2}$ and define block matrices with square blocks of order 8 by

$\gamma_{i}=\left(\begin{array}{cc}A_{i} & 0 \\ 0 & -A_{i}\end{array}\right), \gamma_{6}=\left(\begin{array}{cc}0 & \mathrm{Id} \\ \mathrm{Id} & 0\end{array}\right), \gamma_{7}=\left(\begin{array}{cc}0 & R_{i} \\ -R_{i} & 0\end{array}\right), \gamma_{8}=\left(\begin{array}{cc}0 & R_{j} \\ -R_{j} & 0\end{array}\right), \gamma_{9}=\left(\begin{array}{cc}0 & R_{k} \\ -R_{k} & 0\end{array}\right)$

where $i=1, \ldots, 5$. Here $R_{q}: \mathbb{H} \rightarrow \mathbb{H}$ is right multiplication and we used the same symbol for its natural action on $\mathbb{H}^{2}$.

The subspaces $S_{ \pm} \cong \mathbb{H}^{4}$ are isotropic w.r.t. the canonical symplectic form, which dually pairs them via the standard inner product on $\mathbb{H}^{4}$. Thanks to this and the above Gamma matrices, one easily recovers the Dirac current; here we will just mention that $\kappa\left(S_{ \pm}, S_{ \pm}\right)=\mathbb{R} \boldsymbol{e}_{ \pm}$and $\kappa\left(S_{+}, S_{-}\right)=\mathbb{R}^{9}$.

Lemma 4 The spinorial action of $\widetilde{\mathfrak{h}}$ is given by

$$
\begin{aligned}
& \boldsymbol{e}_{+} \wedge \mathbb{R}^{9}=\left\langle\left(\begin{array}{cc}
0 & \gamma_{i} \\
0 & 0
\end{array}\right) \mid i=1, \ldots, 9\right\rangle, \\
& \mathfrak{s o}\left(\mathbb{E}^{\perp}\right)=\left\langle\left(\begin{array}{cc}
\gamma_{i} \gamma_{j} & 0 \\
0 & \gamma_{i} \gamma_{j}
\end{array}\right) \mid 6 \leq i<j \leq 9\right\rangle, \\
& \mathfrak{u}(1)=\left\langle\left(\begin{array}{cc}
\gamma_{2} \gamma_{3}+\gamma_{4} \gamma_{5} & 0 \\
0 & \gamma_{2} \gamma_{3}+\gamma_{4} \gamma_{5}
\end{array}\right)\right\rangle,
\end{aligned}
$$

where $\gamma_{2} \gamma_{3}+\gamma_{4} \gamma_{5}=-2\left(\begin{array}{c|c|c}\left.\frac{L_{i}}{0}\right|_{0} ^{0} & 0 \\ \hline 0 & \frac{L_{i}}{0}{ }^{0}\end{array}\right)$ as a quaternionic matrix of $\mathbb{H}^{4}$. In particular the subspaces of $S$ given by

$$
\begin{aligned}
& S_{1}^{\prime}=S_{+} \oplus \mathbb{H} \oplus(0) \oplus \mathbb{H} \oplus(0) \\
& S_{2}^{\prime}=S_{+} \oplus(0) \oplus \mathbb{H} \oplus(0) \oplus \mathbb{H}
\end{aligned}
$$

are $\widetilde{\mathfrak{h}}$-stable.

Now $K=\left(\gamma^{\varphi}\right)^{-1}\left(\mathfrak{s t a b}_{\mathfrak{s} \mathfrak{p}(V)}(\varphi)\right)$ clearly contains $\operatorname{Ker} \gamma^{\varphi}$ and since $\Lambda^{1} V \subset \operatorname{Ker} \gamma^{\varphi}$ we may write $K=\operatorname{Ker} \gamma^{\varphi} \oplus C$ for some $C \subset \Lambda^{2} V \oplus \Lambda^{5} V$. Clearly $\operatorname{dim} C=\operatorname{dim} \widetilde{\mathfrak{h}}=16$ and it turns out that 


$$
\begin{aligned}
& \boldsymbol{e}_{23}+\boldsymbol{e}_{45}, 2 \boldsymbol{e}_{13}+\boldsymbol{e}_{36789},-2 \boldsymbol{e}_{12}-\boldsymbol{e}_{26789}, 2 \boldsymbol{e}_{15}+\boldsymbol{e}_{56789},-2 \boldsymbol{e}_{14}-\boldsymbol{e}_{46789}, \\
& \boldsymbol{e}_{45789}+\boldsymbol{e}_{23789}, \boldsymbol{e}_{45689}+\boldsymbol{e}_{23689}, \boldsymbol{e}_{45679}+\boldsymbol{e}_{23679}, \boldsymbol{e}_{45678}+\boldsymbol{e}_{23678} \text { and } \\
& \boldsymbol{e}_{-} \wedge\left\{-2 \boldsymbol{e}_{1}+\boldsymbol{e}_{6789}, \boldsymbol{e}_{4589}+\boldsymbol{e}_{2389}, \boldsymbol{e}_{4579}+\boldsymbol{e}_{2379}, \boldsymbol{e}_{4578}+\boldsymbol{e}_{2378}, \boldsymbol{e}_{4569}+\boldsymbol{e}_{2369}\right. \\
& \left.\boldsymbol{e}_{4568}+\boldsymbol{e}_{2368}, \boldsymbol{e}_{4567}+\boldsymbol{e}_{2367}\right\}
\end{aligned}
$$

are generators of $C$. Using this decomposition one may check that $\odot^{2} S^{\prime} \subset K$ in both cases - in particular $\mathfrak{D} \subset K$ - and that $S^{\prime}$ cannot be enlarged (otherwise one constructs $s \odot t \in \odot^{2} S^{\prime}$ with vanishing Dirac current and such that $\left.\gamma^{\varphi}(s, t) \notin \widetilde{\mathfrak{h}}\right)$.

Corollary 2 The pairs $\left(\varphi, S_{1}^{\prime}\right)$ and $\left(\varphi, S_{2}^{\prime}\right)$ are Lie pairs, and they are maximal.

The Dirac kernel $\mathfrak{D}$ has dimension $\operatorname{dim} \mathfrak{D}=\operatorname{dim} \odot^{2} S^{\prime}-\operatorname{dim} V=289$ in both cases but the two spaces are different; indeed we have

$$
\mathfrak{h}=\gamma^{\varphi}(\mathfrak{D})=\left\{\begin{array}{l}
\widetilde{\mathfrak{h}}=\left(\mathfrak{u}(1) \oplus \mathfrak{s} \mathfrak{D}\left(\mathbb{E}^{\perp}\right)\right) \ltimes\left(\boldsymbol{e}_{+} \wedge \mathbb{R}^{9}\right) \text { if } S^{\prime}=S_{1}^{\prime}, \\
\mathfrak{u}(1) \ltimes\left(\boldsymbol{e}_{+} \wedge \mathbb{E}^{\top}\right) \text { if } S^{\prime}=S_{2}^{\prime},
\end{array}\right.
$$

where $\mathbb{E}^{\top}=\left\langle\boldsymbol{e}_{2}, \boldsymbol{e}_{3}, \boldsymbol{e}_{4}, \boldsymbol{e}_{5}\right\rangle \subset \mathbb{E}$. In the first case

$$
X(v)=\gamma^{\varphi}(\Sigma v) \subset \gamma^{\varphi}\left(\odot^{2} S^{\prime}\right) \subset \widetilde{\mathfrak{h}}=\mathfrak{h}
$$

for any section $\Sigma: V \rightarrow \odot^{2} S^{\prime}$, so we may arrange $X=0$ without loss of generality. It turns out that $\gamma^{\varphi}\left(\odot^{2} S^{\prime}\right) \subset \mathfrak{h}$ in the second case too, so $X=0$. In particular $d \varphi=0$.

Using Gamma matrices, it is straightforward to see

$$
\beta_{\boldsymbol{e}_{+}}=0, \quad \beta_{\boldsymbol{e}_{i}}=\left(\begin{array}{ll}
0 & * \\
0 & 0
\end{array}\right) \quad(i=1, \ldots, 9),
$$

as endomorphisms of $S=S_{+} \oplus S_{-}$, and that $\beta_{\boldsymbol{e}_{-}}$preserves the decomposition of $S$ into eight copies of $\mathbb{H}$. It follows that $\beta_{v}\left(S^{\prime}\right) \subset S^{\prime}$ for all $v \in V$ in both cases.

We are left with the identities involving the curvature tensor $R: \Lambda^{2} V \rightarrow \mathfrak{s o}(V)$. To solve them, we decompose

$$
V=\mathbb{R} \boldsymbol{e}_{+} \oplus \mathbb{R} \boldsymbol{e}_{-} \oplus \mathbb{R} \boldsymbol{e}_{1} \oplus \mathbb{E}^{\top} \oplus \mathbb{E}^{\perp}
$$

and accordingly write $v=v_{+}+v_{-}+v_{1}+v_{\top}+v_{\perp}$ for any $v \in V$. The general strategy to solve (45) is analogous to [8, §4.3], although a tad more involved: we give the final results and briefly comment on how we derived them. In the first and the second case, respectively, we get:

$$
\begin{aligned}
R(v, w)= & -\eta\left(\boldsymbol{e}_{+}, v_{-}\right)\left(\frac{4}{9} w_{1} \wedge \boldsymbol{e}_{+}+\frac{1}{36} w_{\top} \wedge \boldsymbol{e}_{+}+\frac{1}{9} w_{\perp} \wedge \boldsymbol{e}_{+}\right) \\
& +\eta\left(\boldsymbol{e}_{+}, w_{-}\right)\left(\frac{4}{9} v_{1} \wedge \boldsymbol{e}_{+}+\frac{1}{36} v_{\top} \wedge \boldsymbol{e}_{+}+\frac{1}{9} v_{\perp} \wedge \boldsymbol{e}_{+}\right), \\
R(v, w)= & -\frac{1}{4} \eta\left(\boldsymbol{e}_{+}, v_{-}\right) w_{\top} \wedge \boldsymbol{e}_{+}+\frac{1}{4} \eta\left(\boldsymbol{e}_{+}, w_{-}\right) v_{\top} \wedge \boldsymbol{e}_{+},
\end{aligned}
$$

for all $v, w \in V$. A subtle point to obtain equations (56) is that the R.H.S. of 45) makes sense for all $s \in S$ but the action in there is not that of an element of $\mathfrak{s} \mathfrak{D}(V)$. 
Upon restriction to $S^{\prime}$, one may re-absorb to $\Lambda^{2} V$ the contributions coming from the elements in $\operatorname{End}(S) \cong \bigoplus_{0 \leq p \leq 5} \Lambda^{p} V$ with $p \neq 2$, and this is how we arrived at (56). Checking equation (44) is then a direct matter, if tedious, of Clifford identities, as in e.g. [8, Theorem 16].

We recovered two symmetric pp-waves backgrounds that were discovered in [10], one indecomposable and the other decomposable. The proof of the existence comes at the same time with the construction of their transvection superalgebras - they are the filtered deformations of $\mathfrak{a}=\mathfrak{h} \oplus S^{\prime} \oplus V$ determined by $\varphi$-in particular it comes with the knowledge that $N=24$ is the amount of Killing spinors preserved.

Acknowledgements A.S. is grateful to G. Carnovale, F. Esposito and J. Figueroa-O'Farrill for useful discussions. The research of A. S. is supported by the project "Supergravity backgrounds and Invariant theory" at the University of Padova and partly supported by project BIRD179758/17 "Stratifications in algebraic groups, spherical varieties, Kac Moody algebras and Kac Moody groups" and project DOR1717189/17 "Algebraic, geometric and combinatorial properties of conjugacy classes".

\section{References}

1. R. Bryant, Pseudo-Riemannian metrics with parallel spinor fields and vanishing Ricci tensor, in Global analysis and harmonic analysis (Marseille-Luminy 1999), J. P. Bourgignon, T. Branson, O. Hijazi, eds., vol. 4 of Sémin. Congr., pp 53-94. Soc. Math. France, Paris, 2000.

2. S.-J. Cheng, V. G. Kac, Generalized Spencer cohomology and filtered deformations of $\mathbb{Z}-$ graded Lie superalgebras, Adv. Theor. Math. Phys. 2, 1141-1182 (1998).

3. P. de Medeiros, J. Figueroa-O'Farrill, A. Santi, Killing superalgebras for Lorentzian fourmanifolds, J. High Energy Phys. 6, 49 pp (2016).

4. P. de Medeiros, J. Figueroa-O'Farrill, A. Santi, Killing superalgebras for lorentzian sixmanifolds J. Geom. Phys. 132, 13-44 (2018).

5. J. Figueroa-O'Farrill, N. Hustler, The homogeneity theorem for supergravity backgrounds, J. High Energy Phys. 10, 8pp (2012).

6. J. Figueroa-O'Farrill, P. Meessen, S. Philip, Supersymmetry and homogeneity of M-theory backgrounds, Classical Quantum Gravity 22, 207-226 (2005).

7. J. Figueroa-O'Farrill, G. Papadopoulos, Maximally supersymmetric solutions of ten and eleven-dimensional supergravity, J. High Energy Phys. 3, 25pp (2003).

8. J. Figueroa-O'Farrill, A. Santi, Spencer cohomology and 11-dimensional supergravity, Comm. Math. Phys. 349, 627-660 (2017).

9. J. Figueroa-O'Farrill, A. Santi, On the algebraic structures of Killing superalgebras, Adv. Theor. Math. Phys 21, 1115-1160 (2017).

10. J. Gauntlett, C. Hull, pp-waves in 11-dimensions with extra supersymmetry, J. High Energy Phys. 6, 13pp (2002).

11. J. Gauntlett, S. Pakis, The geometry of $D=11$ Killing spinors, J. High Energy Phys. 4, 33 pp (2003).

12. J. Gauntlett, J. Gutowski, S. Pakis, The geometry of $D=11$ null Killing spinors, J. High Energy Phys. 12, 29pp (2003).

13. U. Gran, J. Gutowski, G. Papadopoulos, D. Roest, $N=31, D=11$, J. High Energy Phys. 2, 17 pp (2007).

14. U. Gran, J. Gutowski, G. Papadopoulos, M-theory backgrounds with 30 Killing spinors are maximally supersymmetric, J. High Energy Phys. 3, 38 pp (2010). 
15. T. Harmark, T. Takayanagi, Supersymmetric Gödel universes in string theory, Nuclear Phys. B 662, 3-39 (2003).

16. J. Martinet, Sur les singularités des formes différentielles, Ann. Inst. Fourier (Grenoble) 20, 95-178 (1970).

17. J. Michelson, A pp-wave with 26 supercharges, Classical Quantum Gravity 19, 5935-5949 (2002).

18. V. Patrangenaru, Locally homogeneous pseudo-Riemannian manifolds, J. Geom. Phys. 17, 59-72 (1995).

19. A. Santi, A. Spiro, Super-Poincaré algebras, space-times and supergravities (I), Adv. Theor. Math. Phys. 16, 1411-1441 (2012).

20. A. Santi, A. Spiro, Super-Poincaré algebras, space-times, and supergravities. II., J. Math. Phys. 53, 24 pp (2012).

21. E. Vinberg, A. Elashvili, A classification of the three-vectors of nine-dimensional space, Trudy Sem. Vektor. Tenzor. Anal. 18, 197-233 (1978). 